UNIVERSITÉ DE PICARDIE JULES VERNE

FACULTÉ DE MÉDECINE D’AMIENS

ANNÉE 2019

\title{
État des lieux de l'organisation des biberonneries hospitalières de France en 2018
}

\author{
THÈSE POUR LE DOCTORAT EN MÉDECINE \\ DIPLÔME D'ÉTAT \\ SPÉCIALITÉ PÉDIATRIE \\ $\mathrm{N}^{\circ} 2019-78$
}

PRÉSENTÉE ET SOUTENUE PUBLIQUEMENT

LE 3 SEPTEMBRE 2019

par

Monsieur Mickaël FRÈRE

PRÉSIDENT DU JURY: $\quad$ Monsieur le Professeur TOURNEUX

MEMBRES DU JURY : $\quad$ Monsieur le Professeur DJEDDI

Madame le Professeur DESAILLOUD

Madame le Docteur BOUREL-PONCHEL

DIRECTEUR DE THÈSE : $\quad$ Monsieur le Docteur LEKE 
À mon président de jury,

\section{Monsieur le Professeur Pierre TOURNEUX}

Professeur des Universités-Praticien Hospitalier

(Pédiatrie)

Pôle "Femme - Couple - Enfant"

Vous me faites l'honneur de présider ma thèse.

Merci de m'avoir accompagné dans mon parcours et mon choix d'orientation professionnelle.

Soyez assuré de ma reconnaissance et de mon profond respect. 
À mon jury,

\section{Madame le Professeur Rachel DESAILLOUD}

Professeur des Universités-Praticien Hospitalier

(Endocrinologie, Diabétologie et Maladies Métaboliques)

Vous avez aimablement accepté de juger mon travail.

Je vous remercie chaleureusement de cet honneur.

Soyez assurée de ma reconnaissance et de mon profond respect. 
À mon jury,

\section{Monsieur le Professeur Djamal-Dine DJEDDI}

Professeur des Universités - Praticien Hospitalier

(Pédiatrie)

Responsable du centre d'activité "Pédiatrie médicale et médecine de l'adolescent"

Pôle "Femme - Couple - Enfant"

Je vous remercie d'avoir accepté de juger mon travail.

Vous avez toujours été disponible lorsque j'ai sollicité votre aide, bien que je n'aie pas eu l'opportunité d'effectuer un stage dans votre service.

Soyez assuré de ma reconnaissance et de mon profond respect. 
À mon jury,

Madame le Docteur Emilie BOUREL-PONCHEL

Maître de Conférences des Universités - Praticien Hospitalier

(Pédiatrie)

Exploration fonctionnelle du système nerveux

Vous avez aimablement accepté de juger mon travail.

Soyez assuré de ma reconnaissance et de mon profond respect. 
À mon directeur de thèse,

\title{
Monsieur le Docteur André LEKE
}

\author{
Praticien Hospitalier
}

(Pédiatrie)

Responsable du centre d'activité "Soins intensifs de néonatalogie et unité mère-enfant" Pôle "Femme - Couple - Enfant"

Je tiens à vous remercier tout particulièrement pour tout ce que j'ai appris à vos côtés au cours de mon internat et pour votre profonde gentillesse. Vous m'avez transmis votre passion pour la néonatalogie, dont j’ai décidé de faire ma spécialité.

Je suis très sensible à la confiance que vous m'avez accordée au cours de ces années en me confiant plusieurs travaux dont ce travail de thèse, et pour votre soutien dans mes choix d'orientation professionnelle.

Soyez sûr de ma profonde reconnaissance à votre égard. 


\section{À toute l'équipe médicale et paramédicale du service de soins intensifs de néonatalogie}

et de médecine néonatale, qui ont toujours fait preuve de bienveillance à mon égard et qui ont réussi à accepter mes plaisanteries.

Je remercie tout particulièrement le Docteur André Leke, chef de service, d'avoir cru en moi et de m'avoir accordé toute sa confiance dans les projets qu'il m'a confiés. Merci de m'avoir tant appris, votre expérience et vos connaissances sont précieuses. Je sais que nous avons encore beaucoup de choses à partager.

Merci également à Sabrina, qui a suivi mon parcours avec sa bonne humeur communicative, et à Christel, toujours de bon conseil et disponible. Nous avons partagé de nombreuses gardes dont je garde un excellent souvenir.

Merci à Lucie et à Cécile, sans qui je n'aurais pas pu progresser si vite en salle de naissance.

\section{À tous les médecins qui m'ont accompagné au cours de mes stages et de mes gardes.} L'équipe de pneumopédiatrie et de cardiopédiatrie: Arnaud, Cinthia, Julie, Dorothée, Bérengère, Sabine, Docteur Elizabeth Bourges-Petit, Docteur Amel Mathiron. Les équipes de pédiatrie et de néonatalogie du centre hospitalier de Saint-Quentin: Anne, Claire, Marie, Tiphaine, Docteur Salam Albarazi, Docteur Philippe Dolhem, Epiphanie, Mireille, Tann, Jumana, Docteur Lotfi Hamzaoui, Docteur Souhaïb Al Hawari. L'équipe de réanimation néonatale et pédiatrique, vous m'avez permis d'avoir confiance en moi: Guy, François, Laurent, Yasser, Coralie, Evelyne, Anaïs, Marion, Ghida, Professeur Pierre Tourneux. L'équipe de pédiatrie du centre hospitalier d'Abbeville : Lydia, Rania, Docteur Céline Ségard, Docteur Omar Karzazi.

À toutes les équipes paramédicales : infirmières et infirmiers, puéricultrices et puériculteurs, auxiliaires, aides-soignants, sages-femmes, secrétaires, dont on ne redira jamais l'importance, sans qui rien ne serait possible, et qui méritent notre reconnaissance pour leur dévouement.

Au docteur Marie-Claude Mathieu-Breton, vous avez pris soin de ma santé depuis ma naissance, vous avez suivi mon parcours et ses nombreux tourments. Vous me connaissez mieux que quiconque dans ce métier.

Au docteur Nadine Bouget, vous m'avez accueilli dans votre cabinet pour un stage d'étudiant en médecine générale, vous avez été si gentille avec moi, nous partageons la même passion pour la Bretagne et les $2 \mathrm{cv}$ ! 
À mes co-internes : à ma promotion. Hélène, Loïc, je suis heureux de vous compter parmi mes amis. À Perrine, nous avons tant à partager (et pas que des repas copieux), j'ai hâte de te retrouver à Saint-Quentin. À tous ceux avec qui j'ai partagé un stage ou que j'ai côtoyés au cours de ces quatre années et qui chacun à leur façon ont marqué mon internat : Céline, Lisa, Elise, Romain, Leslie, Lucie, Sarah, Fanny, Sandrine, Cécile, Suzanne, Anne-Sophie, Florinne.

À Clément, pour ton humour sans limites au sens propre comme au sens figuré, pour ton amitié, à Claire et à votre petite Manon, je vous souhaite plein de bonheur dans le foyer douillet que vous vous construisez.

À Camille, depuis le premier jour de notre stage de néonatalogie à Saint-Quentin, tu as pris une place toute particulière dans ma vie. Je ne compte plus nos fous-rires, chaque instant passé à tes côtés est gravé dans ma mémoire. Je ne sais pas comment te remercier pour ton soutien, dans les bons comme dans les mauvais moments. Sache que je serais toujours là pour toi quoi qu'il arrive.

À Laura-Alexandra, depuis notre rencontre au début de nos études de médecine, nous avons construit une amitié sincère et solide. Nous avons choisi des chemins différents, mais finalement pas si éloignés (à moi le début de la vie, à toi son crépuscule). Merci pour ce séjour à Londres mémorable, et j'espère qu'un jour nous parviendrons à faire ce fameux voyage à New York dont je parle tant.

À Alexandru, mon meilleur ami, déjà dix-sept ans d'amitié fidèle malgré la distance, j'ai toujours plaisir à te revoir dès que je le peux, la région dans laquelle tu as choisi de vivre est vraiment magnifique.

À ma famille, que la distance ne me permet pas de voir aussi souvent que je le souhaiterais : mon oncle Michel, ma tante Jacqueline, Colette, mon neveu Antoine, ma cousine Marie-José, Eric, Benoît, Adeline, Laura. À ceux qui nous ont quittés trop tôt : mon oncle Charles, ma tante Nicole, ma tante Maïté.

À mes grands-parents, j' aurais tant aimé que vous soyez là pour partager mon bonheur. Je pense beaucoup à vous. 
À Éric, mon frère, toi qui m'a vu grandir, tu as toujours été si prévenant avec moi, je ne pourrais jamais assez te remercier pour ton soutien sans faille depuis toutes ces années, tu as toujours cru en moi, tu m'as poussé à aller au bout de mes rêves. La Normandie est une belle région, même s'il y pleut quand même beaucoup ! Merci d'être là.

À mes parents, je ne serais jamais devenu ce que je suis aujourd'hui sans vous, vous êtes ce que j'ai de plus précieux au monde, indiscutablement. Je sais que je ne suis pas toujours facile à vivre, que mon caractère est parfois difficile, mais j'espère de tout cœur que je saurais vous rendre fiers. Merci infiniment. Je vous aime plus que tout. 


\author{
Abréviations \\ AFSSA : Agence Française de Sécurité Sanitaire des aliments \\ CHG : Centre Hospitalier Général \\ CHU : Centre Hospitalier Universitaire \\ CLIN : Centre de Lutte contre les Infections Nosocomiales \\ ESPGHAN : European Society for Paediatric Gastroenterology, Hepatology et Nutrition \\ GED : Gestion Electronique des Documents \\ GFHGNP : Groupe Francophone d'Hépatologie-Gastroentérologie et Nutrition Pédiatriques \\ HACCP : Hazard Analysis Critical Point \\ HAS : Haute Autorité de Santé \\ OMS : Organisation Mondiale de la Santé \\ SFP : Société Française de Pédiatrie
}




\section{Table des matières}

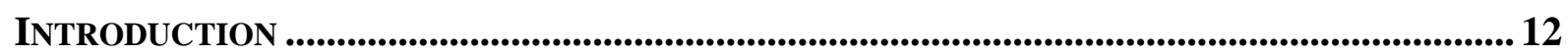

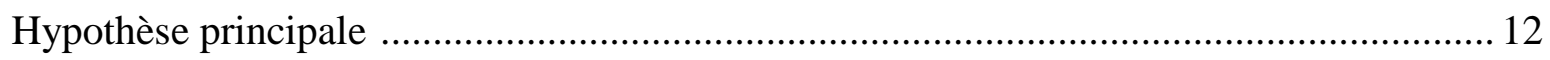

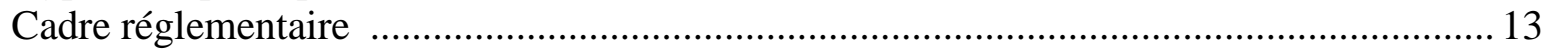

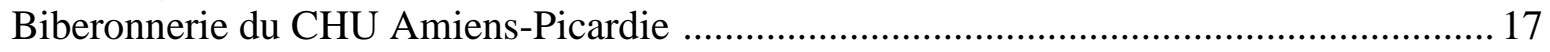

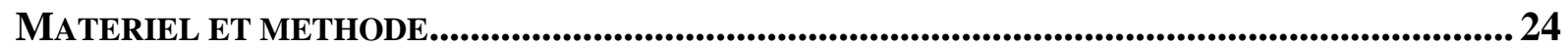

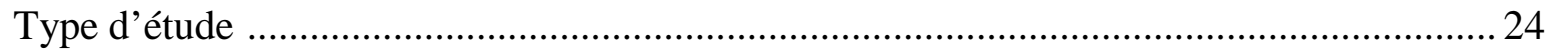

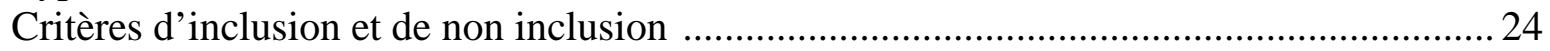

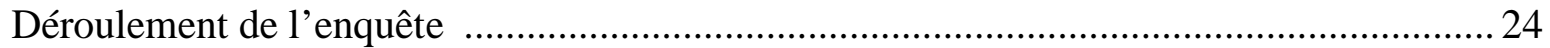

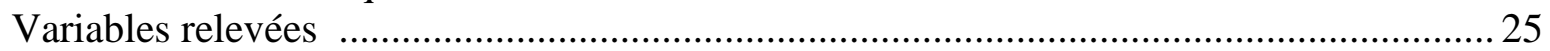

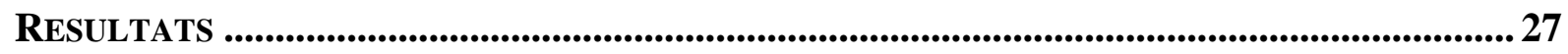

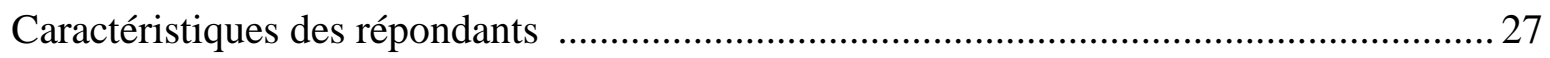

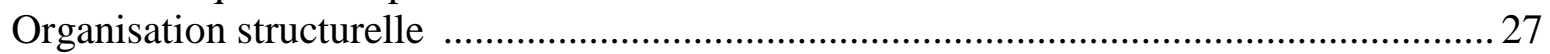

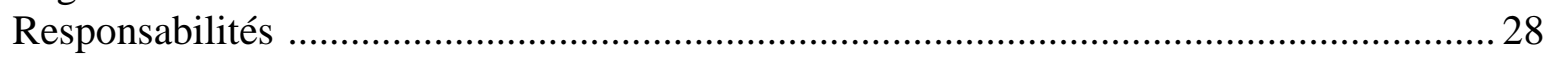

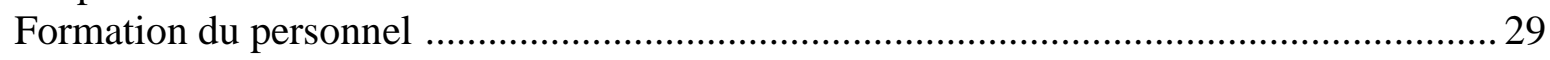

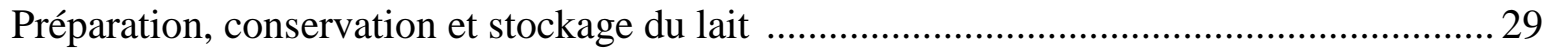

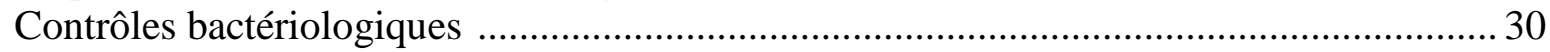

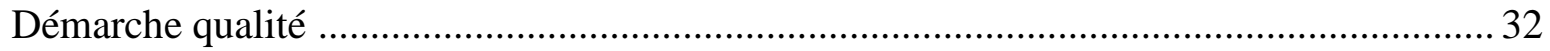

DISCUSSION

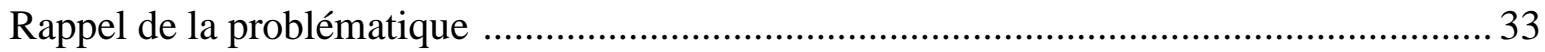

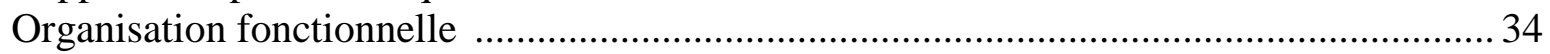

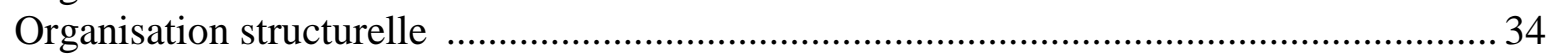

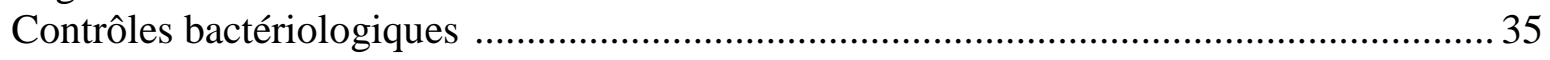

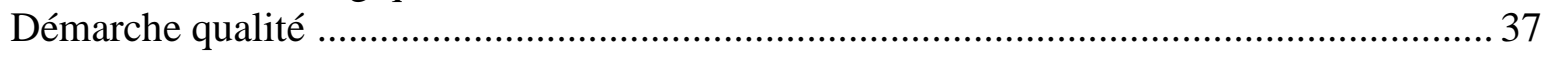

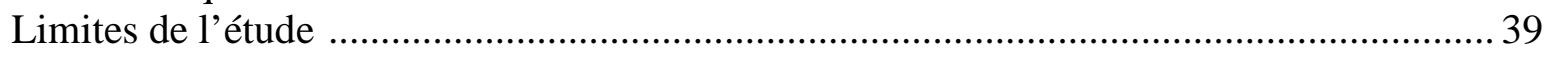

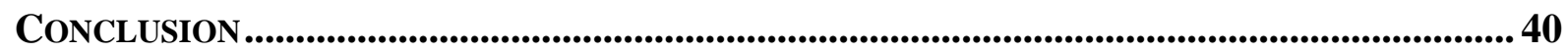

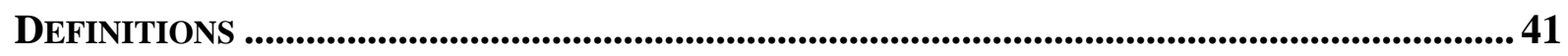

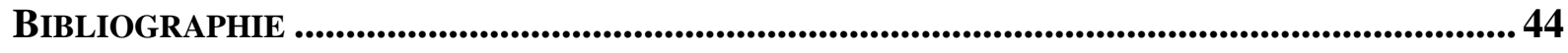

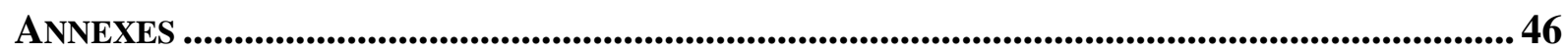

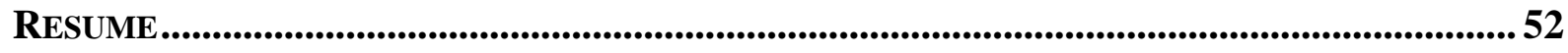




\section{Introduction}

\section{Hypothèse principale :}

Le lait est l'aliment unique et indispensable de tout nouveau-né et nourrisson jusqu'à la diversification alimentaire et reste essentiel à sa croissance au-delà. Bien que l'allaitement maternel soit la solution idéale, il n'est pas toujours possible ou souhaité par les parents. C'est pourquoi ont été élaborés à partir du milieu du XIXème siècle des laits dits «artificiels », actuellement dénommés préparations infantiles, et préparés à partir de lait animal, principalement de vache. Ces préparations répondent à une législation très stricte en matière de composition.

Le pédiatre joue un rôle central pour assurer au quotidien la sécurité alimentaire des jeunes enfants, en particulier les plus fragiles d'entre eux (nourrissons et nouveau-nés prématurés). Il participe à la veille sanitaire en signalant toute infection qui pourrait être secondaire à une contamination bactérienne des préparations lactées.

Dans l'optique de prévenir ce risque infectieux, les autorités sanitaires françaises et européennes ont mis place à partir des années 1990 des référentiels relatifs au stockage et à la délivrance des laits artificiels pour les enfants hospitalisés. La dernière recommandation en date et toujours en vigueur a été publiée par l'Agence Française de Sécurité Sanitaire des Aliments (AFSSA) en juillet $2005^{(1)}$. Néanmoins, nous ne disposons pas de données récentes sur la mise en application de ces recommandations au sein des biberonneries françaises.

Or, la survenue régulière d'épisodes de contaminations des laits infantiles, dont un cas récent de contamination à Salmonella spp sur un site de production français ${ }^{(2)}$ a montré la nécessité d'un contrôle strict et rigoureux du circuit de stockage et de distribution du lait, de sa sortie de la chaîne de production à son administration à l'enfant. Le but de ce travail est donc de réaliser un état des lieux du fonctionnement des biberonneries hospitalières de France au regard des recommandations de l'AFSSA. 


\section{Cadre réglementaire :}

La principale source de recommandations pour la gestion des biberonneries en France a été publiée par l'AFSSA en $2005^{(1)}$.

\section{Les locaux :}

Les locaux type "biberonnerie centrale" font l'objet d'un plan particulier : cheminement du biberon à nettoyer depuis le local de lavage, puis du biberon propre, à partir du local de conditionnement, l'autoclavage, la préparation du biberon, le stockage des biberons. L'architecture de tels locaux reprend les caractéristiques des locaux à environnement protégé (zone 3) comprenant la maîtrise des allées et venues, un point d'eau équipé et le contrôle de qualité de l'eau et de l'air.

Un secteur spécifique doit permettre d'effectuer la préparation, la manipulation, la conservation des biberons et des seringues pour la nutrition entérale. Lorsqu'on ne dispose pas d'une biberonnerie centrale respectant le principe de « la marche en avant », les locaux doivent être séparés en secteur propre et secteur sale. Dans ce cas, il est préférable d'utiliser des biberons «prêts à l'emploi ».

\section{Organisation et formation du personnel :}

Le titulaire de l'autorisation désigne un médecin chargé de l'organisation et du fonctionnement de la biberonnerie pour le site principal et pour ses antennes. Le médecin est assisté d'une sage-femme ou d'un cadre de santé ou d'un infirmier de puériculture.

La qualification est une part importante du bon fonctionnement de la biberonnerie. Le personnel doit accéder à une formation continue à l'hygiène alimentaire, adaptée aux contraintes organisationnelles et aux besoins de chaque poste de travail. Une formation d'adaptation au poste de travail doit être dispensée le cas échéant aux personnels suivants :

- diététiciennes, puéricultrices, ou infirmières et/ou ;

- éducatrices de jeunes enfants ;

- auxiliaires de puériculture (idéalement) ou aide-soignantes et/ou agents hospitaliers ou agents de service intérieur. 
Un responsable de proximité, spécialisé, doit être identifié pour permettre la mise en œuvre effective des bonnes pratiques de préparation et de conservation des préparations nutritionnelles.

Un organigramme nominatif détaillant les différentes activités doit être établi. Les missions et fonctions individuelles doivent être clairement définies et décrites par écrit.

\section{La préparation des biberons :}

Les règles générales sont les suivantes :

- Vérifier la date de péremption de la boîte de lait et de tout produit diététique ajoutée ;

- Effectuer avant l'ouverture de toute boîte de lait un essuyage humide à l'aide d'une solution détergente agréée « contact alimentaire » avec un carré d'essuyage, garder la boîte dans le même local avec sa date d'ouverture mentionnée ;

- Mettre la charlotte et le masque de soins ;

- Se laver les mains ;

- Revêtir la tenue de protection appropriée ;

- Préparer le matériel et les produits, sur le plan de travail nettoyé.

L'eau utilisée pour la préparation des biberons doit être une eau bactériologiquement maîtrisée ou une eau embouteillée réfrigérée, peu minéralisé provenant d'une bouteille ouverte depuis moins de $24 \mathrm{~h}$.

\section{Stockage des biberons :}

Les biberons doivent être stockés, immédiatement après la préparation, dans un réfrigérateur réservé à cet usage, à température $\leq 4^{\circ} \mathrm{C}$, équipé d'un thermomètre minimummaximum et nettoyé et désinfecté de façon hebdomadaire avec un produit désinfectant agrée pour l'usage alimentaire. Le protocole d'entretien du réfrigérateur est établi avec le service d'hygiène, validé par le CLIN. Un étalonnage régulier de l'outil de mesure de la température est nécessaire. Le lait reconstitué et le lait entamé ne doivent pas être conservés au réfrigérateur plus de $24 \mathrm{~h}$. 
Transport :

Il convient d'éviter toute rupture de la chaîne du froid. Que le lait soit réfrigéré à une température inférieure ou égale à $4^{\circ} \mathrm{C}$ ou congelé, il doit être transporté au lieu de consommation dans une glacière ou dans un sac isotherme avec pack eutectique, si la durée de transport est supérieure à 10 minutes.

Réception sur le site de consommation :

Dès l'arrivée sur le site de consommation, vérifier que les biberons de lait ont été correctement identifiés (nom et prénom de l'enfant, date et heure du 1er recueil), et placer les biberons, soit dans une enceinte réfrigérée à une température inférieure ou égale à $4^{\circ} \mathrm{C}$, soit au congélateur.

\section{Consommation du biberon :}

Les biberons ne doivent être sortis de l'enceinte réfrigérée de conservation qu'immédiatement avant leur utilisation.

Tout biberon sorti de l'enceinte réfrigérée doit être consommé dans un délai d'1 heure. La remise en température doit avoir lieu dans les minutes précédant la consommation au chauffe-biberon à sec. En pratique hospitalière, il n'est pas conseillé d'utiliser des systèmes de réchauffement utilisant de l'eau (bain-marie, chauffe-biberon à eau).

L'utilisation du four à micro-ondes est totalement proscrite (risque de brûlures et diminution de la qualité nutritionnelle du lait).

\section{Contrôles - réglementation:}

La biberonnerie doit disposer d'un système de management de la qualité adapté, sous la responsabilité du médecin responsable ou d'une personne compétente qu'il aura désignée. Le système qualité mis en place doit être évalué lors de réunions périodiques avec la direction. Un bilan annuel permettra d'évaluer les actions entreprises et de définir les nouveaux objectifs. 
Différents contrôles peuvent être mis en œuvre pour maîtriser l'hygiène et assurer la qualité microbiologique des biberons :

- Prélèvement sur les surfaces et le matériel de préparation ;

- Analyse microbiologique de l'eau ;

- Analyse microbiologique des biberons reconstitués.

Les contrôles microbiologiques et physico-chimiques de l'eau doivent être effectués en fonction du système d'assurance-qualité mis en place dans l'établissement (fréquence minimale trimestrielle).

Idéalement la biberonnerie doit être équipée d'un système de gestion informatisé certifié.

Entretien des locaux et du matériel :

Quelle que soit la structure et selon la réglementation, un plan de nettoyage et de désinfection est défini par écrit.

L'automatisation de la procédure de nettoyage et de désinfection des matériels de préparation et des biberons justifie que cette procédure soit effectuée par un procédé de désinfection thermique.

Le nettoyage et la désinfection doivent être effectués par des appareils automatiques, qui sont de deux types :

- les laveurs-désinfecteurs professionnels ;

- les lave-vaisselle. 


\section{Biberonnerie du CHU Amiens-Picardie :}

\section{Historique :}

Le CHU Amiens-Picardie s'est doté d'une biberonnerie unique en 2003, à l'époque sur le site de l'hôpital nord. Avant 2003, chaque service de pédiatrie disposait de son espace de stockage et de préparation du lait. Dans le cadre de la construction du nouveau CHU sud et du regroupement de l'ensemble des services de pédiatrie sur ce site en 2015, une nouvelle biberonnerie unique a été conçue. Elle est associée à un lactarium dont les missions sont la promotion de l'allaitement maternel, la collecte, la conservation et la distribution du lait de mère. Géographiquement, elle est adjacente aux unités de réanimation néonatale, de soins intensifs de néonatalogie et de médecine néonatale. La biberonnerie du CHU Amiens-Picardie a passé une convention avec le centre hospitalier d'Abbeville et assure la fourniture du lait pour ses services de pédiatrie et de néonatalogie.

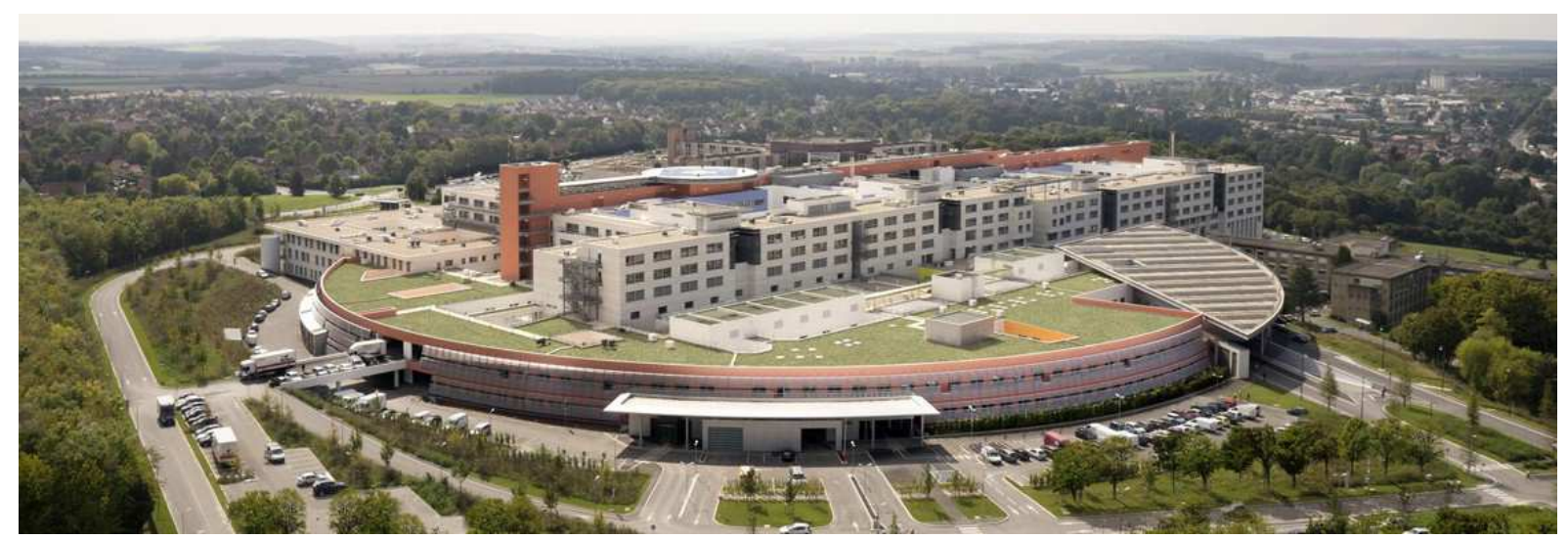

Figure 1: CHU Amiens-Picardie, site sud, inauguré en 2015

\section{Responsabilités et ressources humaines :}

La biberonnerie du CHU Amiens-Picardie est rattachée au pôle femme-couple-enfant dont le chef de pôle est le Professeur Patrick Berquin. Elle est sous la responsabilité du service de soins intensifs de néonatalogie et de médecine néonatale, et son référent médical est le Docteur André Leke, chef de service. Au niveau paramédical, la biberonnerie est sous la responsabilité de la cadre supérieure de santé Mme Béatrice Longo. Quatre auxiliaires de puériculture sont chargées de la préparation du lait, sous la coordination d'une infirmière. (cf. Annexe $\mathrm{n}^{\circ} 2$ : organigramme). 
Les prescriptions de lait sont assurées en premier lieu par les médecins responsables ou les internes. Elles peuvent être déléguées aux puéricultrices et infirmières sous la responsabilité de l'équipe médicale. Pour les préparations spéciales, elles peuvent être réalisées par les diététiciennes des secteurs de pédiatrie, Mme Laurie Rousseau et Mme Marie-Pascale Fremaux. Ces prescriptions sont intégralement informatisées via le logiciel DxC@ re et sont donc totalement tracées.

\section{Organisation :}

La biberonnerie du CHU Amiens-Picardie a été conçue selon le principe de la marche en avant, avec un flux du lait en sens unique : stockage puis préparation et enfin délivrance aux unités de soins, sans retour en arrière possible (cf. Annexe $\left.\mathrm{n}^{\circ} 3\right)$. L'accès à la biberonnerie se fait par un sas équipé d'un lavabo permettant le lavage des mains. Pour entrer dans la zone de préparation du lait, il est obligatoire de revêtir un équipement de protection à usage unique comprenant une blouse, une charlotte et des gants. Le lait en poudre est stocké à température ambiante, tandis que le lait en nourrettes prêtes à l'emploi est stocké en chambre froide à $4^{\circ} \mathrm{C}$. Le matériel de préparation du lait (pots et fouets) est lavé dans un lave-vaisselle puis envoyé en stérilisation par autoclave.

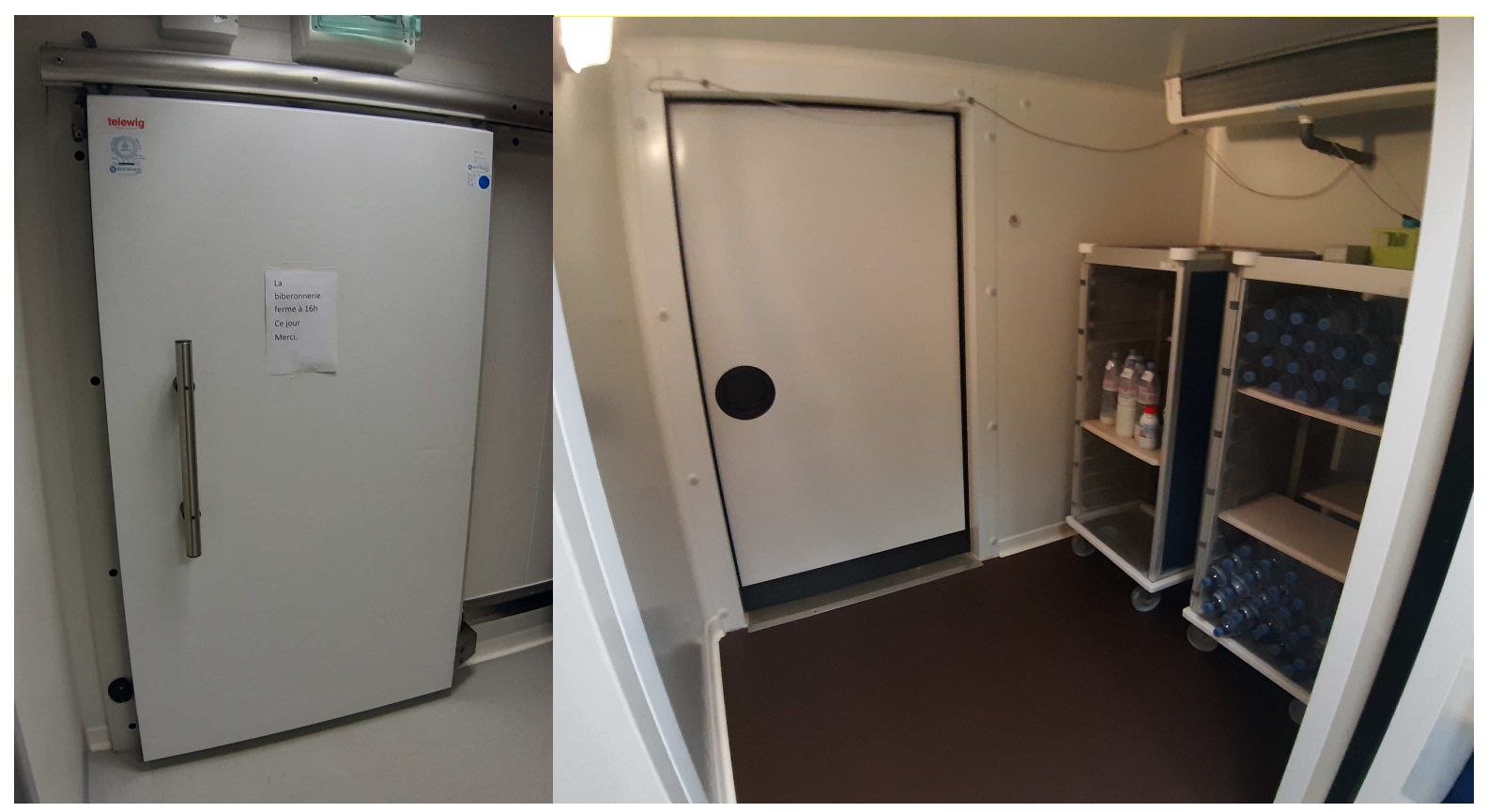

Figure 2: chambre froide 


\section{Circuit du lait :}

Le lait peut être délivré en nourrettes prêtes à l'emploi, en biberons ou en seringues selon les besoins de l'enfant: nourrettes ou biberon pour les enfants autonomes sur le plan alimentaire, seringues pour les nouveau-nés sous alimentation entérale par sonde nasogastrique. Les nourrettes prêtes à l'emploi sont délivrées directement dans les services. Les biberons et le seringues sont stériles et à usage unique.

Une fois préparé, le lait sort de la biberonnerie via un sas de distribution à sens unique. Son acheminement dans les services se fait à l'aide d'un chariot de transport réfrigéré avec plaques eutectiques permettant le respect de la chaine du froid en maintenant le lait à une température de $4^{\circ} \mathrm{C}$. Ces mêmes chariots sont utilisés pour le transport du lait vers le centre hospitalier d'Abbeville.

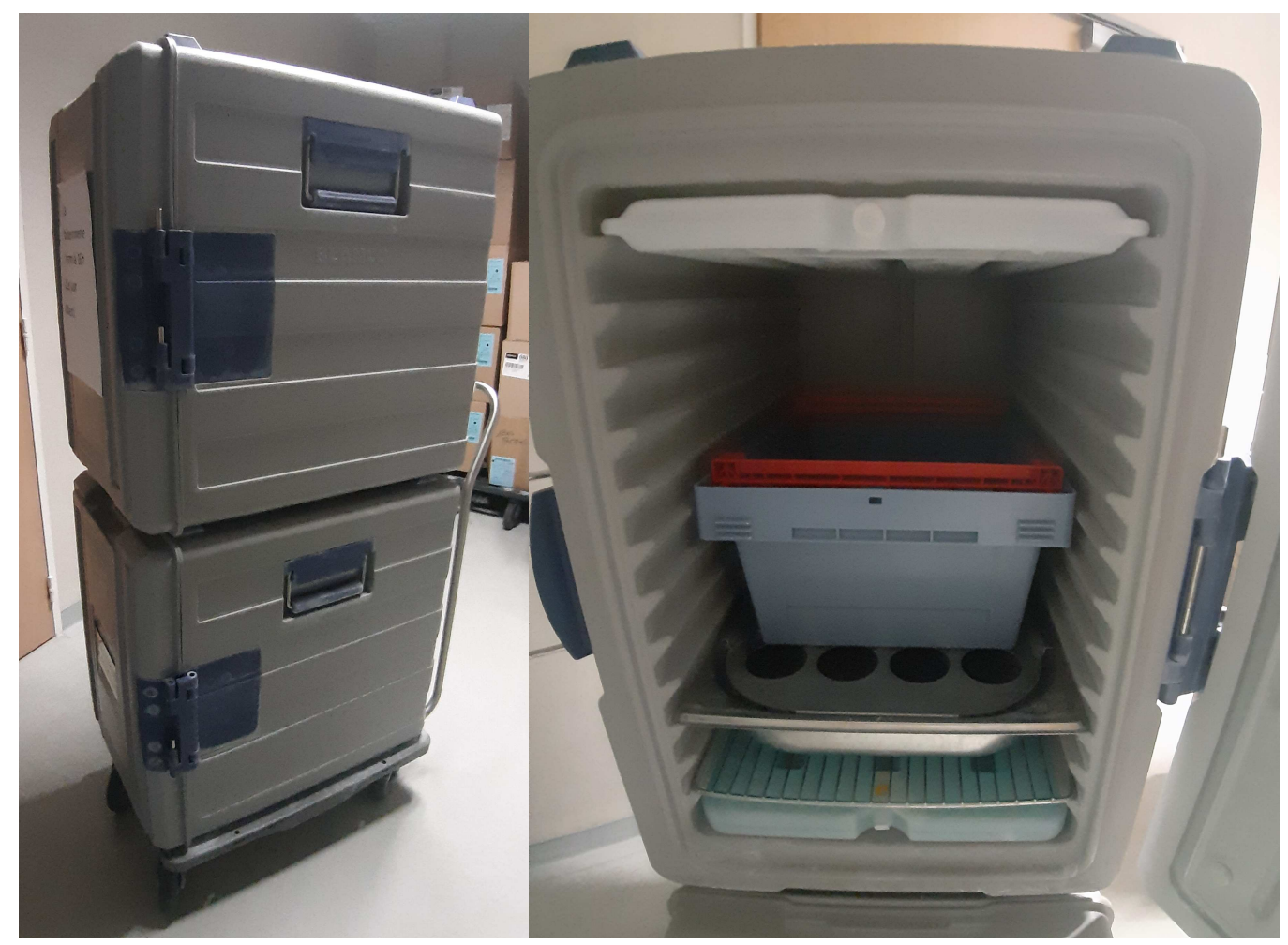

Figure 3 : chariot de transport du lait avec plaques eutectiques

Une fois le lait délivré, les chariots de transports sont désinfectés et lavés dans une pièce dédiée avant toute réutilisation. 
Contrôles bactériologiques :

Les contrôles bactériologiques sur les laits et poudres sont externalisés : les prélèvements sont réalisés par la société Silliker SAS et envoyés pour analyse au laboratoire Mérieux (cf. Annexe n4). Ces contrôles portent sur :

- Les matières premières (poudres): 1 prélèvement par semaine à jours variables sur une boite entamée de lait ;

- Les produits finis:

○ Préparations "standards" : 1 prélèvement par semaine à jours variables pour un type de lait ;

○ Préparations spécifiques à base de lait en poudre : 1 prélèvement par semaine à jours variables sur la préparation ayant eu le plus grand nombre de manipulations ;

- Après transport (dans le service demandeur avant mise au réfrigérateur) :

○ 1 prélèvement par semaine sur les préparations standards ;

- 2 prélèvements par semaine sur les préparations spécifiques ;

- Après conservation 24 heures dans le réfrigérateur du service de soins :

- 1 prélèvement par semaine sur les préparations standards ;

○ 2 prélèvements par semaine sur les préparations spécifiques ;

Les germes spécifiquement recherchés par le laboratoire sont :

- Microorganismes aérobies à $30^{\circ} \mathrm{C}$;

- Coliformes à $30^{\circ} \mathrm{C}$ : si positif, recherche et identification de Cronobacter spp et Escherichia coli ;

- Staphylococcus aureus ;

- Clostridium perfringens ;

- Salmonella spp;

- Levures et moisissures.

Le coût annuel des analyses bactériologiques est estimé à environ $12000 €$. Pour l'année 2018, il est détaillé dans le tableau 1. 


\begin{tabular}{|l|r|r|r|r|}
\hline SERVICE BIBERONNERIE & \multicolumn{1}{|c|}{$\begin{array}{c}\text { Montant } \\
\text { HT }\end{array}$} & T.V.A & \multicolumn{1}{c|}{$\begin{array}{c}\text { Montant } \\
\text { TTC }\end{array}$} \\
\hline 1. Analyses biberonnerie & 468 unités & $9671,48 €$ & $20 \%$ & $11605,78 €$ \\
\hline 2. Audits hygiène biberonnerie & 1 unité & $198,00 €$ & $20 \%$ & $237,60 €$ \\
\hline $\begin{array}{l}\text { 3. Autres prestations: audits hygiène } \\
\text { offices alimentaires biberonnerie }\end{array}$ & & & & \\
\hline Analyses sous 24 heures & 1 unité & $100,00 €$ & $20 \%$ & $120,00 €$ \\
\hline Recherche Escherichia coli O157 & 1 unité & $33,50 €$ & $20 \%$ & $40,20 €$ \\
\hline Confirmation Escherichia coli O157 H7 & 1 unité & $19,88 €$ & $20 \%$ & $23,86 €$ \\
\hline Sérotypage Escherichia coli O157 H7 & 1 unité & $96,00 €$ & $20 \%$ & $115,20 €$ \\
\hline Entérotoxine Staphylococcique & 1 unité & $160,00 €$ & $20 \%$ & $192,00 €$ \\
\hline Sérotypage Salmonelle & 1 unité & $84,00 €$ & $20 \%$ & $100,80 €$ \\
\hline
\end{tabular}

Tableau 1: coût des contrôles bactériologiques du lait

\section{Démarche qualité :}

La démarche qualité au sein de notre biberonnerie est sous la responsabilité du service hygiène et du service qualité du CHU Amiens-Picardie. Dans le cadre de cette démarche, toutes les procédures décrivant le fonctionnement de la biberonnerie sont réactualisés régulièrement, et disponibles en version informatique sur la GED de l'hôpital. Elles ont été établies selon les recommandations du CLIN Paris-Nord de $1999^{(12)}$ relatives aux conditions de préparation et au circuit des biberons pour l'alimentation du nourrisson en collectivité pédiatrique. Ces procédures sont révisées par les référents médicaux et paramédicaux de la biberonnerie en conformité avec la réglementation. Pour s'assurer de la bonne mise en application des procédures, des visites de risque avec audit sont réalisées annuellement. Elles permettent de mettre en évidence des pistes d'amélioration et d'élaborer des actions correctives : c'est la démarche HACCP.

Par ailleurs, le CHU Amiens-Picardie a engagé un programme d'informatisation du secteur biberonnerie lactarium. Cette informatisation est actuellement en cours de mise en place au sein du lactarium. Le logiciel de gestion sélectionné est MO-Lactarium ${ }^{\circledR}$ de la société Mediware. Ce logiciel doit permettre la traçabilité complète du circuit des préparations infantiles, de leur stockage à leur délivrance aux unités de soins. En cas d'alerte sanitaire, il devient ainsi possible de remonter facilement aux lots incriminés et de connaître les enfants qui les auraient éventuellement reçus. L'informatisation du secteur biberonnerie interviendra dans un second temps. 
Enfin, la démarche qualité impose d'assurer une traçabilité stricte des prescriptions de lait, des produits utilisés et des températures de stockage. Les prescriptions sont tracées à l'aide du logiciel de prescription DxC@re. Les numéros de lots des produits utilisés sont répertoriés dans un classeur spécifique. En cas de contamination d'un lot de lait, cette traçabilité doit nous permettre de savoir exactement quel enfant a reçu ce lait. Les températures des réfrigérateurs, des congélateurs et de la chambre froide sont tracées à l'aide d'un système électronique de surveillance nommé SPY ${ }^{\circledR}$.

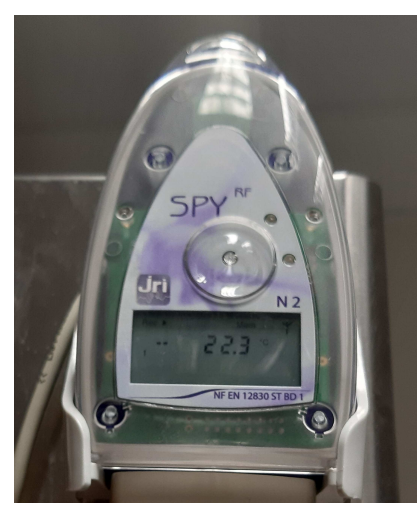

Figure 4 : système de traçabilité informatisé de la température SPY ${ }^{\circledR}$ 


\section{Objectif de l'étude}

\section{Objectif principal :}

- Réaliser un état des lieux de l'organisation et du fonctionnement des biberonneries dans les centres hospitaliers de France en 2018. 


\section{Matériel et méthode}

\section{Type d'étude :}

Nous avons réalisé une étude des pratiques professionnelles, descriptive, transversale, multicentrique, consultant les biberonneries hospitalières de France.

\section{Critères d'inclusion et de non inclusion :}

\subsection{Critères d'inclusion :}

Nous avons inclus toutes les biberonneries hospitalières françaises, en CHU et en centre hospitalier général.

\subsection{Critères de non inclusion :}

Nous n'avons pas inclus les biberonneries qui ne relèvent pas d'un établissement de santé, en particulier les structures d'accueil de jeunes enfants (crèches).

\section{Déroulement de l'enquête :}

Pour réaliser cette étude, nous avons élaboré un questionnaire en ligne envoyé à tous les hôpitaux de France équipés d'une biberonnerie via trois canaux :

- Association des lactariums de France ;

- Société Française de Pédiatrie (SFP);

- Groupe Francophone d'Hépatologie-Gastroentérologie et Nutrition Pédiatriques (GFHGNP).

L'étude se concentre sur la gestion du lait artificiel dans les établissements de santé, et n'inclut donc pas la préparation et la conservation du lait maternel qui relève de la responsabilité du lactarium. 
Ce questionnaire a été structuré pour apprécier la mise en application des recommandations de l'AFSSA de 2005. Il porte sur les éléments clés de la recommandation :

- Responsabilités au sein de la biberonnerie ;

- Formation du personnel ;

- Organisation structurelle des locaux ;

- Préparation, conservation et transport des biberons ;

- Contrôles bactériologiques ;

- Entretien des locaux et du matériel ;

- Assurance qualité.

Le questionnaire complet est détaillé en annexe 1.

Notre questionnaire a été soumis via un formulaire en ligne à partir de septembre 2018. Plusieurs relances ont été réalisées au bout de 3 et 6 mois auprès des établissements n'ayant pas répondu.

\section{Variables relevées :}

Les données suivantes ont été relevées :

\section{Données générales :}

- Type d'établissement de santé : CHU ou CHG

- Nom de l'établissement

- Date d'ouverture de la biberonnerie

\section{Données structurelles :}

- Type de biberonnerie : unique ou multiple ;

- Type d'organisation spatiale : marche en avant ou secteur propre/sale, proximité avec les unités de soins. 
Données organisationnelles :

- Service de rattachement de la biberonnerie ;

- Equipe référente : référent médical, référent paramédical, missions ;

- Ressources humaines : agents dédiés, programmes de formation ;

- Personnes habilitées à la prescription du lait ;

- Informatisation.

\section{Données liées au circuit du lait :}

- Tenue utilisée par les agents pour la préparation du lait ;

- Règles d'hygiène appliquées pour la préparation du lait ;

- Mode de stockage des matières premières ;

- Durée maximale de stockage ;

- Température de stockage, traçabilité ;

- Eau utilisée pour la préparation des laits en poudre ;

- Conditions de transport dans les unités de soins.

\section{Données bactériologiques :}

- Référentiel utilisé ;

- Laboratoire chargé des contrôles bactériologiques, coût ;

- Périodicité des contrôles ;

- Germes recherchés ;

- Conduite à tenir en cas de contamination ;

- Entretien du matériel. 


\section{Résultats}

\section{Caractéristiques des répondants :}

26 centres hospitaliers ont répondu à notre étude, répartis sur l'ensemble du territoire français. Sur ces 26 établissements, 20 (77\%) sont des CHU, soit un taux de réponse de 66.7\% (20/30). 6 centres hospitaliers généraux ont participé à l'enquête (cf. figure 1).

\section{Centre hospitalier universitaire $\quad$ Centre hospitalier général}

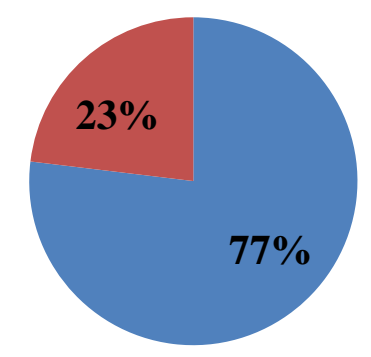

Figure 4: type d'établissements de santé ayant répondu à l'étude

\section{Organisation structurelle :}

25 biberonneries sont de type unique, desservant tous les services de pédiatrie. 9 d'entre elles sont situées à proximité immédiate des services de soins.

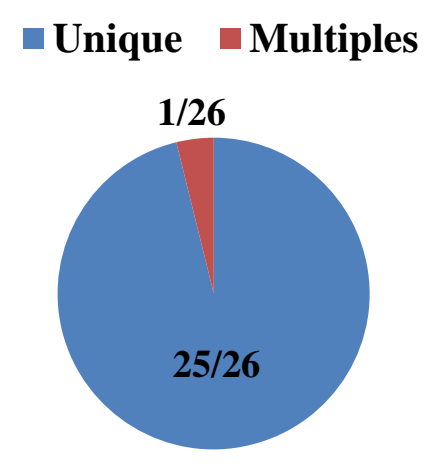

Figure 5: types de biberonneries hospitalières

L'organisation selon le principe de la marche en avant (préparation par étapes successives sans possibilité de retour en arrière) est respectée par $69.2 \%$ des établissements. Une séparation en secteur propre/secteur sale est prévue par les établissements restants. 


\section{Responsabilités :}

Les biberonneries hospitalières ayant répondu à notre enquête sont majoritairement rattachées au service de néonatalogie (12/26) suivi du lactarium (4/26) (cf. figure 3). Une biberonnerie est sous la responsabilité du service des urgences pédiatriques. Enfin, une biberonnerie fonctionne en totale autonomie.

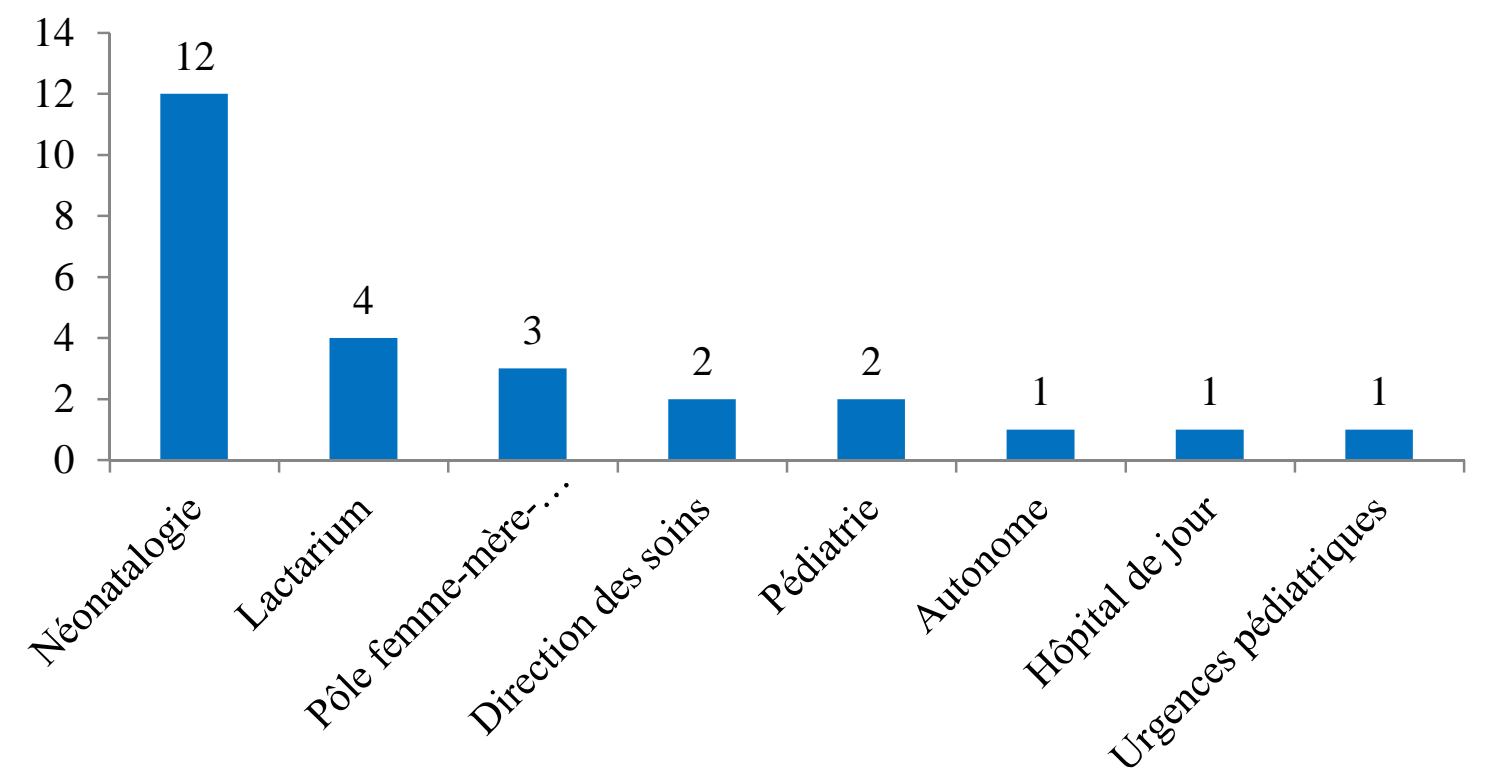

Figure 6: services de rattachement de la biberonnerie

Le référent médical est un médecin néonatologiste pour 14 biberonneries et un gastropédiatre pour 4 d'entre elles. Il est à noter que 4 biberonneries n'ont pas de référent médical clairement identifié. 4 établissements n’ont pas pu répondre à cette question.

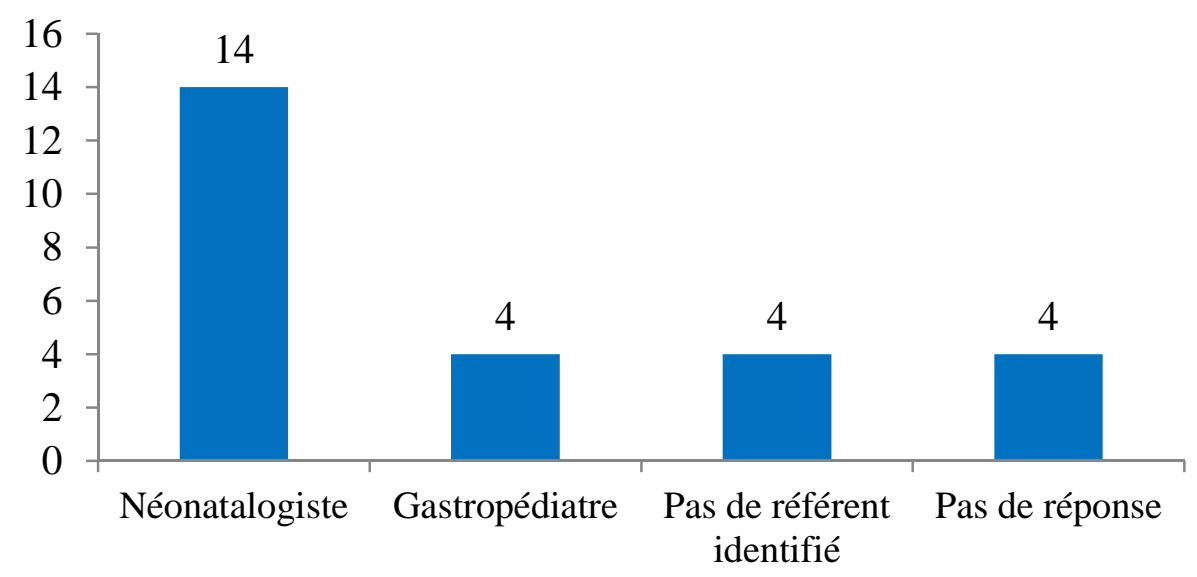

Figure 7: référent médical 
Le référent paramédical est un cadre de santé dans près de 3/4 des biberonneries (19/26), ou une puéricultrice pour 4 unités. Une biberonnerie a pour référent paramédical une auxiliaire de puériculture.

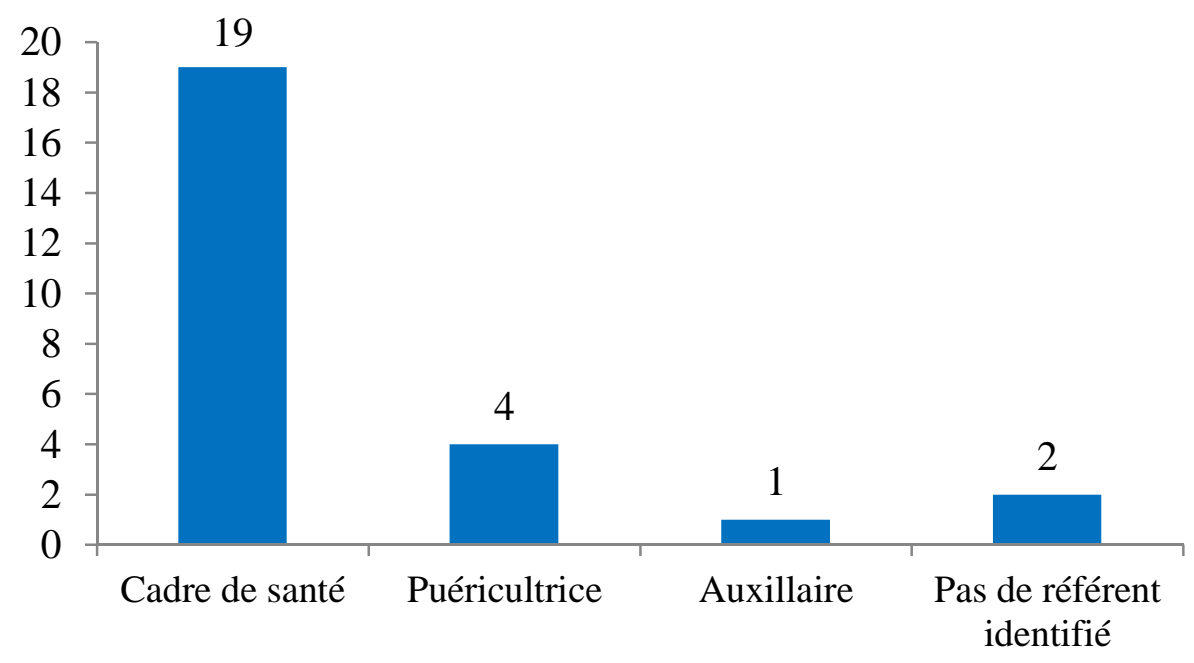

Figure 8: référent paramédical

Les prescriptions de lait sont assurées essentiellement par les médecins et les internes (100\%). Elles peuvent également être réalisées par les diététiciens dans 4 biberonneries et par les puéricultrices pour 3 d'entre elles.

\section{Formation du personnel :}

23 Biberonneries disposent d'agents dédiés exclusivement à la préparation des biberons. Ces agents bénéficient tous d'une formation hygiène mise à jour de manière régulière.

\section{Préparation, conservation et stockage du lait :}

Tous les agents ont à leur disposition une tenue adaptée à usage unique pour la préparation du lait.

La température de stockage du lait artificiel est rigoureusement tracée dans $73.1 \%$ des cas. Seules 3 biberonneries désinfectent le matériel de stockage (réfrigérateurs et chambres froides) de manière hebdomadaire. 
L'acheminement du lait dans les unités de soins se fait au moyen d'un système réfrigéré dans 22 établissements (16 chariots réfrigérés avec plaques eutectiques et 6 glacières). Deux biberonneries transportent le lait à température ambiante. Deux établissements n'ont pas répondu.

\section{Contrôles bactériologiques :}

Les résultats de notre enquête nous montrent que les référentiels utilisés pour l'organisation des contrôles bactériologiques au sein des biberonneries sont très diverses. 6 biberonneries se basent sur les recommandations du guide de bonnes pratiques, tandis que 2 établissements utilisent les recommandations du CLIN. 7 biberonneries ont établi à un référentiel local. Pour 2 d'entre elles il n'a pas été possible de connaître le référentiel et 9 biberonneries n'ont pas répondu à cette question.

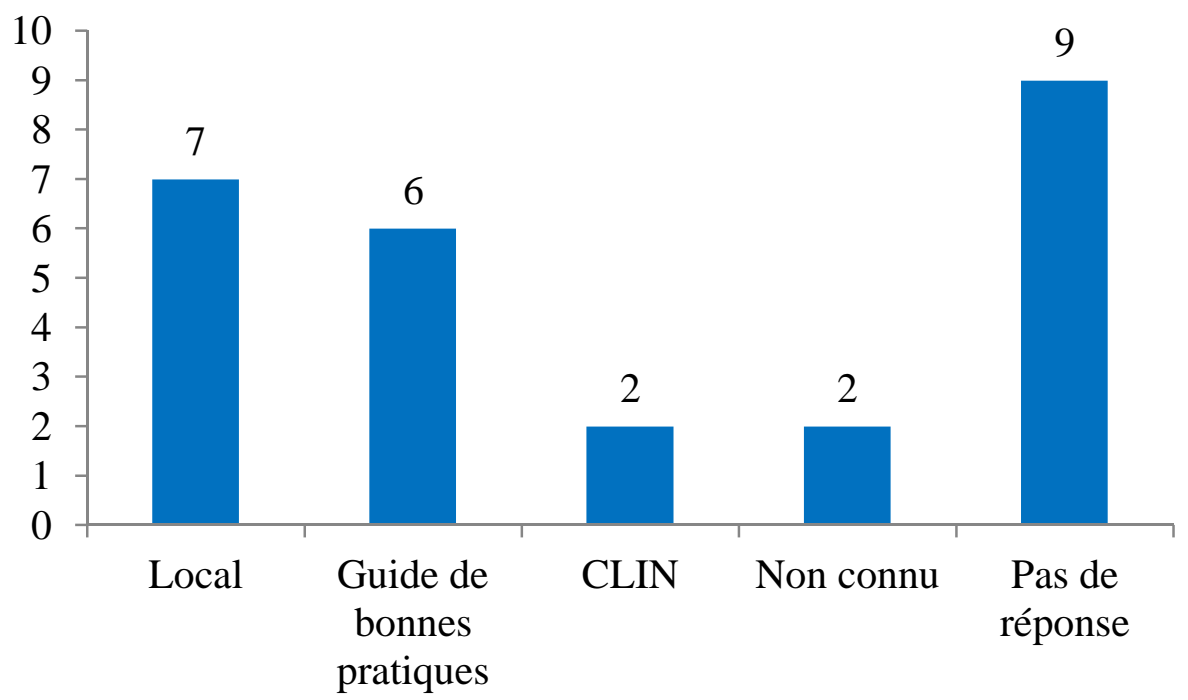

Figure 9: référentiel utilisé pour les contrôles bactériologiques

L'intervalle de réalisation des contrôles bactériologiques est également très hétérogène : hebdomadaire pour 4 établissements, 1 mensuel, 1 bimestriel, 1 trimestriel et 1 semestriel. 1 biberonnerie déclare ne pas réaliser de contrôles bactériologiques systématiques sur le lait. Il n'a pas été possible d'avoir de réponse pour 17 biberonneries. Pour les répondants, les germes recherchés sont les entérobactéries (Escherichia coli et Cronobacter spp), Listeria monocytogenes, Staphylococcus aureus, Salmonella spp. et Bacillus cereus. 


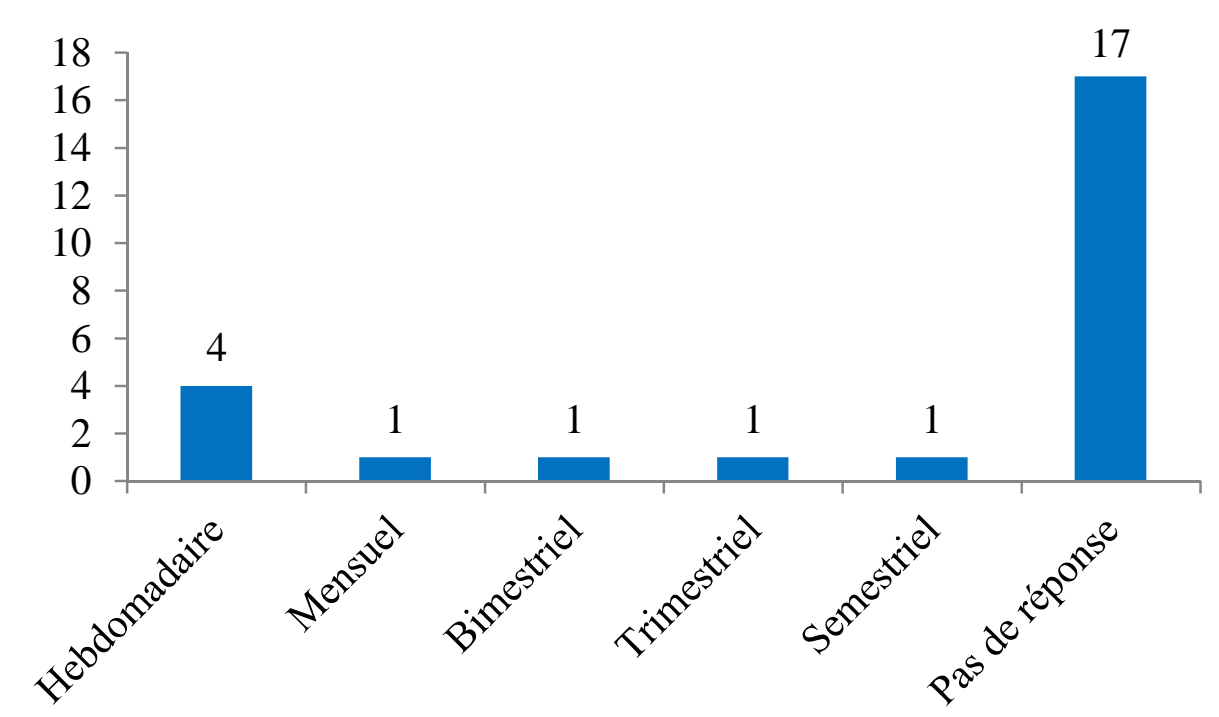

Figure 10 : intervalle de réalisation des contrôles bactériologiques

La majorité des biberonneries font réaliser leurs analyses bactériologiques en interne, par le laboratoire d'hygiène, le laboratoire de bactériologie ou le CLIN. Deux biberonneries font appel à des laboratoires extérieurs à l'hôpital. Nous n'avons pas eu de réponse pour six établissements.

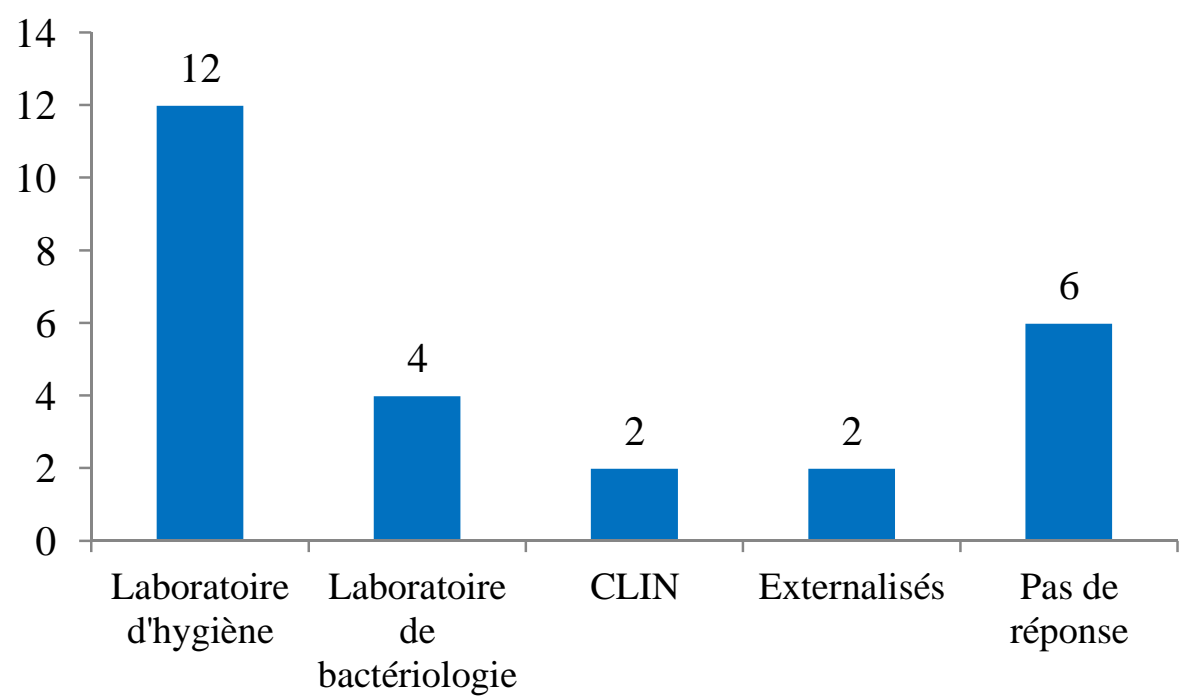

Figure 11 : laboratoire réalisant les analyses bactériologiques

Concernant l'eau utilisée pour la préparation du lait, elle est contrôlée sur le plan bactériologique dans 5 établissements. Il s'agit de biberonneries qui utilisent l'eau du robinet. Les autres biberonneries ont recours à de l'eau en bouteilles. 


\section{Démarche qualité :}

20 établissements (76.9\%) déclarent avoir mis en place une démarche qualité de type HACCP. Cela passe par la rédaction de protocoles (22 biberonneries), accessibles à l'ensemble du personnel concerné (20 biberonneries). Le référent qualité est formellement identifié dans 19 biberonneries. Il peut s'agir du médecin référent, de la cadre de santé ou du pharmacien qualité pour un des établissements.17 biberonneries $(64.5 \%)$ sont équipées d'un système de gestion informatisée du lait. La majorité des biberonneries ont opté pour le logiciel Mo Lactarium ${ }^{\circledR}$ (12/17) développé spécifiquement pour cela.

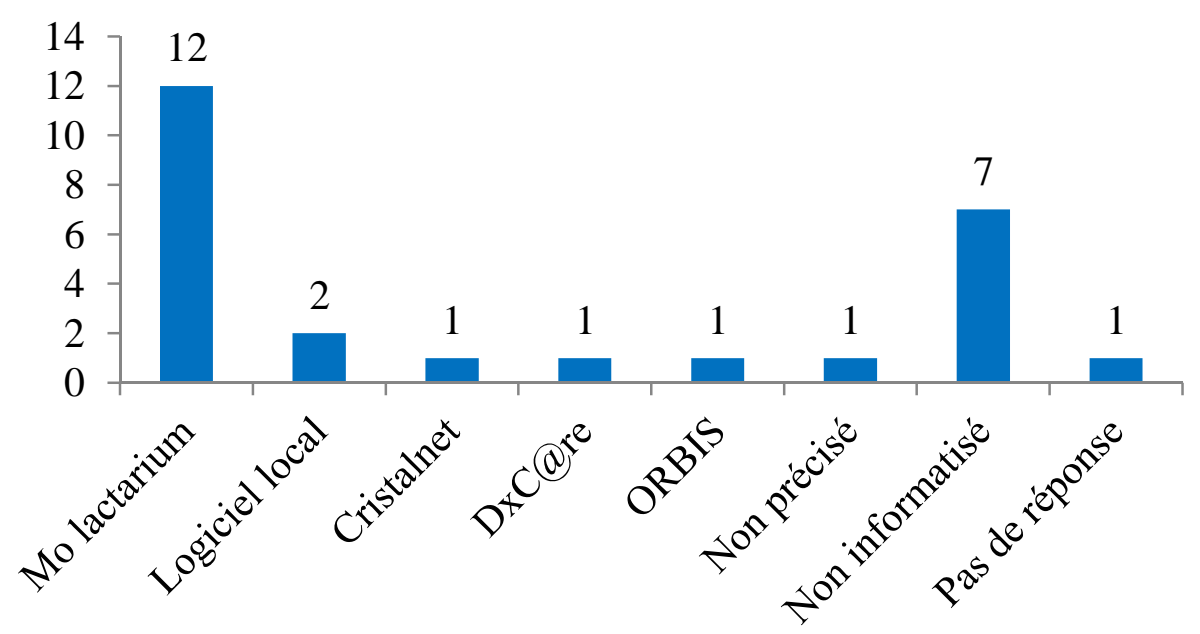

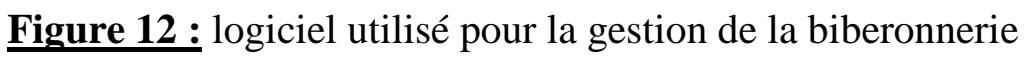




\section{Discussion}

\section{Rappel de la problématique :}

La préparation et le stockage des préparations infantiles doivent répondre à des normes strictes en termes d'hygiène. Ces préparations peuvent se présenter sous deux formes : liquide prête à l'emploi ou poudre à reconstituer. Si le risque de contamination existe dans les deux cas, il est prépondérant avec les poudres à reconstituer qui nécessitent plusieurs manipulations et l'apport d'eau ${ }^{(3)}$. Par ailleurs, une étude américaine de Steele et al en 2008 a montré que ce risque est 24 fois plus important si le lait est reconstitué au lit du patient au lieu d'une pièce

dédiée ${ }^{(4)}$. Les bactéries les plus fréquemment en cause sont connues : Cronobacter $\operatorname{spp}^{(5,6,7)}$ et Salmonella spp. Ces deux germes sont connus pour être responsables d'infections néonatales sévères, potentiellement mortelles.

C'est pourquoi, face à ce risque majeur, l'OMS a énoncé en $2007^{(8)}$ les trois mesures principales permettant de le réduire :

- Diluer la poudre de lait dans une eau chauffée à $70^{\circ} \mathrm{C}$ minimum afin d'inactiver Cronobacter spp ;

- Consommer le lait rapidement après sa préparation ;

- Stocker le lait reconstitué à moins de $5^{\circ} \mathrm{C}$.

Des recommandations similaires ont été établies en Europe et au Canada, dont les recommandations de l'AFSSA datant de $2005^{(1)}$. Cependant, elles ne font pas consensus. En effet, un désaccord existe avec l'ESPGHAN concernant la température de l'eau, l'ESPGHAN rappelant en $2004^{(9)}$ qu'une température excessive peut faire perdre jusqu'à $25 \%$ de ses nutriments au lait, principalement des vitamines, et qu'elle expose par ailleurs au risque de brûlures graves. Il n'existe pas à ce jour d'étude qui a comparé ces deux recommandations.

En tant que pédiatres, nous sommes confrontés quotidiennement à la nécessité d'assurer la sécurité alimentaire des enfants hospitalisés dans nos unités. Récemment, plusieurs affaires de contamination bactériennes des préparations infantiles, dont une sur un site de production français en $2018^{(2)}$ ont relancé les discussions autour de la sécurité sanitaire des aliments conçus pour les très jeunes enfants, qui se doivent d'être irréprochables. Si nous 
n'avons pas le contrôle sur la fabrication des préparations infantiles, nous avons néanmoins une responsabilité majeure dans leur reconstitution et leur administration à nos patients, par l'intermédiaire de nos biberonneries hospitalières. L'ensemble de ce contexte nous a poussés à nous interroger sur la pertinence de nos pratiques et nous a encouragés à savoir ce qui se fait plus globalement en France, dans le but d'aller vers une harmonisation des pratiques.

\section{Organisation fonctionnelle :}

Nos interrogations portaient d'abord sur l'organisation fonctionnelle des biberonneries. Sur ce sujet, la recommandation est claire: chaque biberonnerie doit être sous la responsabilité d'un référent médical, qui peut déléguer certaines missions à un référent paramédical, mais qui reste garant du respect des bonnes pratiques et de la sécurité sanitaire. Notre enquête montre que cette recommandation est parfaitement respectée. Le référent paramédical est dans la grande majorité des cas (73\%) cadre de santé, encadrant également la néonatalogie, et faisant donc le lien entre la préparation et l'administration du lait. $46 \%$ des biberonneries sont sous la responsabilité du service de néonatalogie. Il est à noter qu'une des biberonneries est rattachée aux urgences pédiatriques de son établissement. Même si ce choix peut surprendre, les recommandations ne précisent pas de quel service la biberonnerie hospitalière doit dépendre.

\section{Organisation structurelle :}

Sur le plan structurel, toutes les biberonneries à l'exception d'une sont de type unique. Le stockage et la préparation du lait pour l'ensemble des services de pédiatrie sont ainsi centralisés en un seul lieu. Cette organisation doit permettre d'assurer une sécurité sanitaire maximale puisqu'elle limite les intermédiaires. Par ailleurs, avoir plusieurs biberonneries expose au risque de disparités dans le respect des procédures, engendre un coût supérieur en nécessitant plus de matériel (notamment de stockage) et complexifie la réalisation des contrôles bactériologiques.

Le principe de la marche en avant est plutôt bien appliqué. Ce principe est simple : la préparation du lait est décomposée en plusieurs étapes successives avec impossibilité de retour à une étape antérieure. Il est à la base des démarches qualitatives de l'hygiène, et doit permettre d'assurer qu'aucun matériel ou produit souillé ne peut entrer en contact avec du 
matériel ou des produits sains. Sa bonne application nécessite une organisation logique des locaux avec des pièces dédiées à chaque tâche, ce qui a été réalisé au CHU Amiens-Picardie : sas d'entrée, pièce de stockage des matières premières, pièce de préparation, pièce de stockage du lait prêt à consommer, sas de transfert vers les unités de soins. Le matériel utilisé et les déchets générés à chaque étape doivent être systématiquement éliminés de la chaîne avec une séparation secteur propre/secteur sale claire. Le matériel doit être décontaminé.

\section{Contrôles bactériologiques :}

La directive européenne $\mathrm{CE} \mathrm{n}^{\circ} 2073 / 2005$ de $2005^{(10)}$, relative aux critères microbiologiques applicables aux denrées alimentaires a établi des critères de sécurité bactériologique pour les laits en poudre à destination des nourrissons (cf. tableau 2). Ces critères portent sur les germes à haut risque d'infection néonatale sévère, dont elle impose une absence totale de détection dans les prélèvements :

- Listeria monocytogenes ;

- Salmonella spp ;

- Entérotoxine de Staphylococcus aureus;

- Cronobacter spp.

\begin{tabular}{|c|c|}
\hline Listeria monocytogenes & Absence dans 25g \\
\hline Salmonella & Absence dans 25g \\
\hline Entérotoxines staphylococciques & Pas de détection dans 25g \\
\hline Cronobacter & Absence dans $10 \mathrm{~g}$ \\
\hline
\end{tabular}

Tableau 2 : critères de sécurité des laits en poudre selon la directive CE n ${ }^{\circ} 2073 / 2005^{(10)}$

Les recommandations de l'AFSSA imposent la réalisation de contrôles bactériologiques sur le lait artificiel. En revanche, elles ne précisent pas comment et par qui doivent être réalisés les contrôles. Il s'agit d'une différence majeure avec la réglementation qui s'impose aux lactariums, qui recueillent et conservent le lait maternel. En effet, la décision du code de la santé publique du 3 décembre 2007 définissant les règles de bonnes pratiques ${ }^{(11)}$ est très stricte sur les critères bactériologiques de non-conformité du lait maternel et sur le moment de leur réalisation (cf. tableau 3). 
Analyses à effectuer sur les sous-lots et les lots :

Lorsque le sous-lot constitué par les dons d'une même donneuse, fait l'objet d'un contrôle bactériologique systématique, l'examen consiste en une numération de la flore totale aérobie sur gélose au sang après incubation pendant 24 heures à $37^{\circ} \mathrm{C}$;

Les sous-lots sont déclarés non conformes si la flore totale aérobie après 24 heures d'incubation à $37^{\circ} \mathrm{C}$, est égale ou supérieure à $10^{6}$ bactéries par millilitre.

Les sous-lots conformes sont regroupés en lots et chaque lot fait l'objet des contrôles bactériologiques suivants avant d'être conditionné en flacons et pasteurisé :

- Une numération de la flore totale aérobie sur gélose au sang après 48 heures d'incubation à $37^{\circ} \mathrm{C}$;

- Une recherche et une numération des germes Staphylococcus coagulase positif sur milieu de Chapman après 48 heures d'incubation à $37^{\circ} \mathrm{C}$.

Les lots sont déclarés non conformes si :

- La flore aérobie est égale ou supérieure à $10^{6}$ bactéries par millilitre ;

- Ou si le nombre de germes Staphylococcus coagulase positif est égal ou supérieur à $10^{4}$ bactéries par millilitre.

\section{Analyses à effectuer sur les lots uniquement :}

Lorsque les sous-lots ne sont pas systématiquement contrôlés, le lot fait l'objet des mêmes contrôles bactériologiques avant d'être conditionné en flacons et pasteurisés.

Dans ce cas, les lots sont déclarés non conformes si :

- La flore aérobie est égale ou supérieure à $10^{5}$ bactéries par millilitre ;

- Ou si le nombre de germes Staphylococcus coagulase positif est égal ou supérieur à $10^{4}$

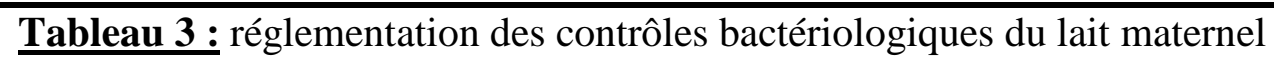


En conséquence, les résultats de notre enquête ont montré que l'organisation des contrôles bactériologiques du lait artificiel est très différente d'un centre à un autre. Le CHU Amiens-Picardie a fait le choix de contrôles hebdomadaires sur les laits en poudres et les laits liquides stockés dans sa biberonnerie, avec envoi des prélèvements à un laboratoire extérieur (Mérieux). Mais plusieurs centres réalisent des contrôles mensuels voire trimestriels. Par ailleurs, de nombreux centres n'ont pas répondu précisément à cette question malgré plusieurs relances. Il apparaît donc que les pratiques sont loin d'être harmonisées sur l'ensemble du territoire français.

\section{Démarche qualité :}

Les biberonneries hospitalières manipulent des préparations infantiles qui sont des denrées alimentaires. Elles sont donc soumises aux mêmes règles d'hygiène strictes que les entreprises du secteur de l'agro-alimentaire et de la restauration, et c'est dans cette optique que la notion de démarche qualité et de méthode HACCP a été introduite par la directive européenne 93/43/CEE le 14 juin $1993^{(13)}$, transcrite dans le droit français par l'article 5 de l'arrêté du 29 septembre 1995 ${ }^{(14)}$. La méthode HACCP (Hazard Analysis Critical Point) a été élaborée par la NASA (National Aeronotics and Space Administration) aux Etats-Unis au milieu du XXème siècle pour assurer la sécurité alimentaire des astronautes. Elle a pour objectif d'éliminer ou de réduire à un niveau acceptable tout risque biologique, chimique ou physique, par une analyse des dangers puis la détermination de points critiques à maîtriser.

Elle peut être décomposée en 7 étapes :

1. Procéder à une analyse des dangers ;

2. Déterminer les points critiques pour la maîtrise ;

3. Fixer le ou les seuil(s) critiques(s) ;

4. Mettre en place un système de surveillance des limites critiques permettant de s'assurer que les points critiques maîtrisent effectivement les dangers ;

5. Déterminer les mesures correctives à prendre lorsque la surveillance révèle qu'un CCP donné n'est pas maîtrisé ;

6. Appliquer des procédures de vérification afin de confirmer que la méthode HACCP fonctionne efficacement ;

7. Constituer un dossier dans lequel figurent toutes les procédures et tous les relevés concernant ces principes et leur mise en application (traçabilité). 


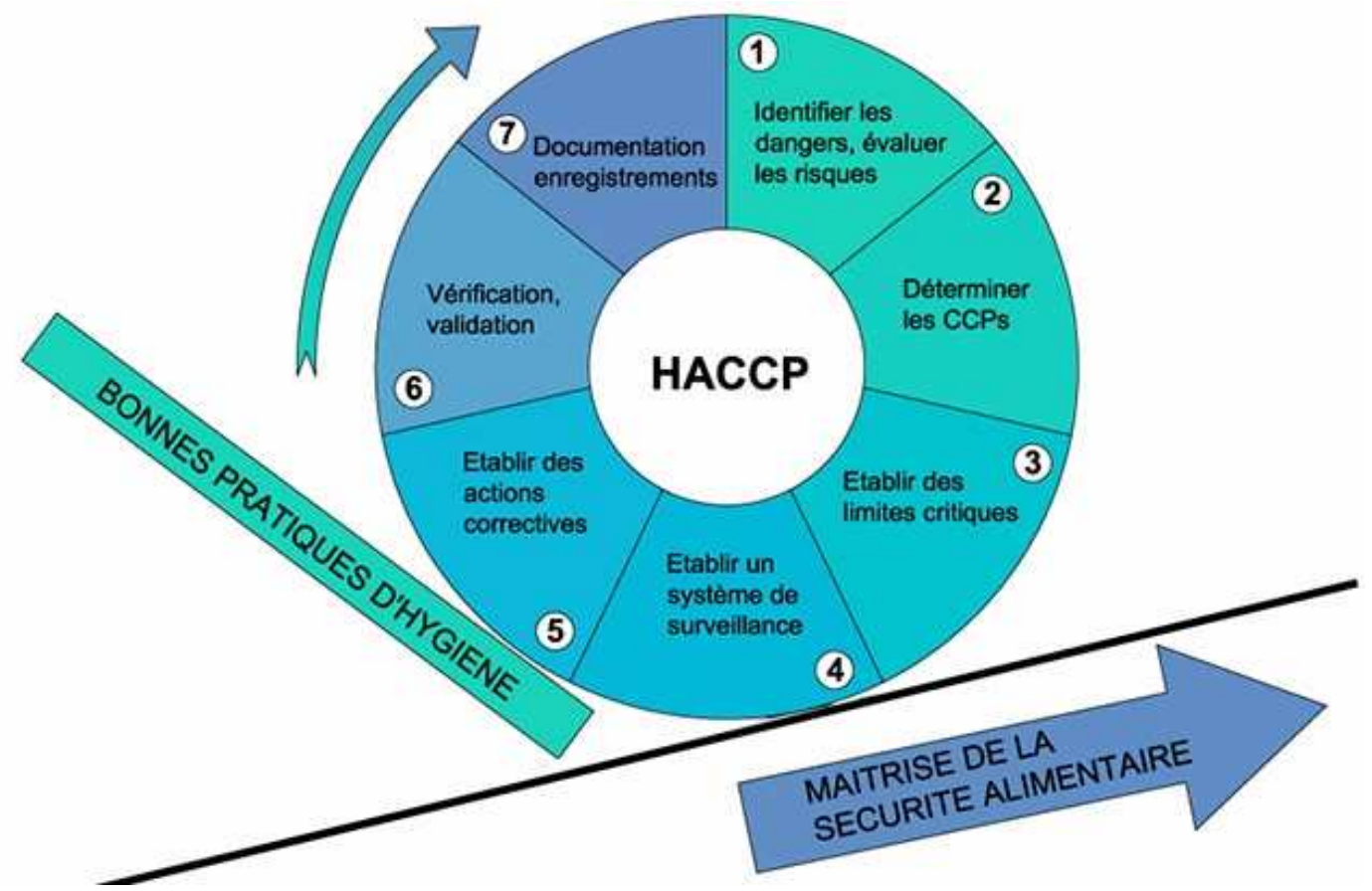

Figure 13: Principe de la démarche qualité HACCP

La mise en place de cette démarche passe par la description de toutes les tâches effectuées par la rédaction de procédures claires, la définition du rôle de chacun au sein de la biberonnerie par l'élaboration de fiches de poste, la mise en place d'un système de surveillance et de mesures correctives permettant de corriger les écarts constatés par cette surveillance.

Au sein d'une biberonnerie hospitalière, le risque microbiologique est présent à de nombreuses étapes et faire l'objet d'un point critique devant être contrôlé :

- Les agents : ils doivent être munis de tenues adaptées, avoir une hygiène des mains rigoureuse, et un même agent ne doit pas opérer à la fois sur le circuit propre et le circuit sale ;

- Le circuit du matériel : le matériel propre et le matériel sale ne doivent jamais entrer en contact, ce qui implique la mise en place de circuits séparés ;

- Stockage des matières premières et du lait préparé : la température de stockage doit être scrupuleusement surveillée ;

- Préparation du lait et eau utilisée : chaque étape de préparation du lait peut présenter un risque de contamination bactérienne, en particulier la manipulation 
des poudres et l'ajout de l'eau qui à défaut d'être embouteillée doit être contrôlée bactériologiquement ;

- Conditions de transport du lait vers les unités de soins : la chaîne du froid doit être respectée ;

- Entretien des locaux : la désinfection des locaux et des surfaces doit être régulière et tracée.

L'identification de ces points critiques mène ensuite à la rédaction de procédures décrivant toutes les tâches réalisées dans la biberonnerie et les moyens de surveiller que ces tâches sont correctement effectuées. Cela conduit à la réalisation d'audits permettant d'évaluer le respect des procédures. En cas de non-respect, on parle de non-conformité. Des actions correctives doivent être mises en place pour lever ces non-conformités. Enfin, un audit de contrôle doit vérifier l'efficacité des actions correctives.

\section{Limites de l'étude :}

La première limite de notre étude est qu'il s'agit d'une enquête sur questionnaire, ce qui engendre inévitablement un biais de déclaration. Cela est particulièrement vrai pour les questions relatives à des sujets pouvant être qualifiés de sensibles, comme celles concernant les contrôles bactériologiques. Plusieurs centres n'ont pas répondu ou ont répondu de manière vague à ces questions, ce qui peut interroger sur la fiabilité de ces réponses. On peut de ce fait émettre l'hypothèse que seuls les centres étant sûrs de la qualité de leurs contrôles bactériologiques ont répondu exhaustivement.

La deuxième limite est liée au type de répondants. Si nous avons obtenu un bon taux de réponse auprès des CHU (20/30 soit 66.7\%), il n'en va pas de même avec les centres hospitaliers généraux, dont seuls six ont répondu. Il n'est pas possible de connaître leur taux de réponse, car il n'a pas été possible de connaître le nombre exact de biberonneries en France. En conséquence, cette prédominance de CHU limite la représentativité de notre enquête. On peut en effet s'attendre à ce que les CHU soient attentifs au respect des recommandations en tant que centres de références. Il n'est en revanche pas évident cela puisse être le cas dans tous les hôpitaux de périphéries, par manque de moyens notamment. 


\section{Conclusion}

L'objectif principal de notre étude était de faire un état des lieux de l'organisation et du fonctionnement des biberonneries hospitalières françaises, à la recherche d'une prise en compte optimale du risque sanitaire lié au lait artificiel. À l'issue de notre enquête, nous avons ainsi pu mettre en lumière de nombreux points positifs, ainsi que des points d'amélioration possible.

Parmi les points positifs, nous avons constaté que les responsabilités sont parfaitement identifiées au sein des biberonneries qui nous ont répondu. Toutes ont désigné un référent médical et un référent paramédical, dont les missions ont été clairement définies. Par ailleurs, les biberonneries sont conçues dans un souci de respect des règles d'hygiène, avec respect du principe de la marche en avant, séparation en secteur propre/secteur sale, tenues adaptées à usage unique, stérilisation du matériel et formations hygiènes régulières du personnel. Les biberonneries suivent en cela les mesures appliquées dans le domaine de la restauration.

Concernant les pistes d'amélioration, le point le plus délicat qui ressort de notre enquête porte sur les contrôles bactériologiques. Si tous les établissements recherchent effectivement les germes responsables d'infections graves chez le nouveau-né et le nourrisson (en particulier Cronobacter spp et Salmonella spp), l'organisation de ces contrôles est très différente d'un centre à l'autre. Les référentiels et les intervalles de contrôles sont en effet très variables, d'autant qu'aucune réglementation ne fixe clairement de critères pour le lait artificiel au contraire du lait maternel.

En conclusion, au vu des résultats de notre étude, il s'avère nécessaire d'harmoniser les pratiques au sein des biberonneries, plus particulièrement sur la question des contrôles bactériologiques du lait, pour bénéficier de la meilleure assurance qualité possible et d'assurer la sécurité nutritionnelle des enfants dans nos hôpitaux. 


\section{Définitions}

- Assurance qualité : large concept qui couvre tout ce qui peut, individuellement ou collectivement, influencer la qualité d'un produit. Elle représente l'ensemble des mesures prises pour s'assurer que le lait contrôlé ait la qualité requise pour l'usage auquel il est destiné.

- Audit : examen méthodique, indépendant et documenté, dont le but est de déterminer si les procédures et les résultats relatifs à la qualité sont de nature à permettre l'atteinte des objectifs fixés.

- Contrôle : ensemble d'opérations visant à déterminer la conformité du produit aux exigences spécifiques.

- Décontamination : opération permettant de supprimer les micro-organismes indésirables. C'est le premier traitement à effectuer sur les objets et matériels souillés dans le but d'éliminer les micro-organismes et de faciliter le nettoyage et la stérilisation ultérieurs.

- Délivrance : mise à disposition sur prescription médicale en vue de l'administration à un nouveau-né.

- Distribution : fourniture du lait à un service de soins d'un établissement de santé ou à un autre lactarium.

- Dossier de lot : comporte toutes les informations relatives à la préparation, au traitement, au conditionnement et aux contrôles du lot préparé. Il porte le numéro du lot de lait.

- Enregistrement : document présentant des résultats obtenus ou la preuve de la réalisation d'une activité.

- Informatisation : mise en place d'un système informatique comprenant la saisie des données, le traitement électronique et la sortie d'informations destinées à être utilisées à des fins de contrôle automatique, de bilans, ou de traçabilité. 
- Libérer/libération : processus qui permet de lever la quarantaine soit des dons de lait collectés pour leur traitement, soit des lots de lait traités pour leur délivrance ou leur distribution, après décision de leur conformité.

- Lot : quantité définie de lait préparé en une opération ou en plusieurs opérations, telle qu'elle puisse être considérée comme homogène. Sauf cas particulier, il est souhaitable que la taille du lot soit limitée au volume de 4 litres.

- Lyophilisation : procédé de transformation d'une solution liquide en poudre.

- Maintenance : ensemble d'actions maintenant ou rétablissant une entité dans un état lui permettant d'accomplir une fonction attendue.

- Management de la qualité : activités coordonnées visant à diriger et à contrôler une organisation en matière de qualité.

- Mode opératoire : description détaillée de la façon de réaliser une activité.

- Non-conformité : défaut observé ou rapporté, relatif aux activités et aux produits.

- Pasteurisation : méthode de stérilisation par la chaleur discontinue permettant d'inhiber les micro-organismes dans un produit peu contaminé.

- Personne habilitée : personne possédant les qualifications requises par les lois et règlements et reconnue par son responsable fonctionnel, capable d'accomplir les tâches qui lui sont confiées.

- Procédure : manière spécifiée d'effectuer une activité.

- Qualification : opération destinée à démontrer qu'un appareil ou matériel fonctionne correctement et donne les résultats attendus. Pour le personnel, la qualification correspond à la formation acquise et requise par la réglementation en vigueur. Elle est entretenue par la formation interne ou externe à laquelle le personnel est tenu de participer. 
- Quarantaine : isolement physique ou par d'autres moyens efficaces des dons collectés et des lots de laits traités dans l'attente d'une décision sur leur conformité ou leur nonconformité.

- Retour : renvoi d'un flacon de lait au lactarium, que le produit présente ou non un défaut de préparation.

- Sous-lot : mélange de dons provenant d'une même donneuse et destinés à entrer dans la composition d'un lot de lait.

- Stérilisation : opération qui a pour but de supprimer d'un objet ou d'un produit tout micro-organisme vivant qui le contamine.

- Traçabilité : possibilité, à partir d'une identification enregistrée, de retrouver l'historique, l'utilisation ou la localisation d'un lait à toutes les étapes de sa collecte, de sa préparation et de sa distribution. La traçabilité d'un lot de lait désigne l'établissement du lien entre la donneuse, le don, le lot délivré ou distribué, l'acheminement et le devenir.

- Validation : opération permettant d'apporter la preuve que les résultats escomptés ont été obtenus dans des conditions techniques satisfaisantes. 


\section{Bibliographie}

1. Agence Française de Sécurité des Aliments. [Recommendations for hygiene in the preparation and maintenance of bottles for feeding infants]. Arch Pediatr 13 Spec No 1, $7-42$ (2006).

2. Jones, G. et al. Outbreak of Salmonella enterica serotype Poona in infants linked to persistent Salmonella contamination in an infant formula manufacturing facility, France, August 2018 to February 2019. Eurosurveillance 24, (2019).

3. Turck, D. Safety Aspects in Preparation and Handling of Infant Food. Annals of Nutrition and Metabolism 60, 211-214 (2012).

4. Steele, C. \& Short, R. Centralized Infant Formula Preparation Room in the Neonatal Intensive Care Unit Reduces Incidence of Microbial Contamination. Journal of the American Dietetic Association 108, 1700-1703 (2008).

5. Kalyantanda, G., Shumyak, L. \& Archibald, L. K. Cronobacter Species Contamination of Powdered Infant Formula and the Implications for Neonatal Health. Frontiers in Pediatrics 3, (2015).

6. Hamrin, P. \& Hoeft, B. Quality Control throughout the Production Process of Infant Food. Annals of Nutrition and Metabolism 60, 208-210 (2012).

7. Henry, M. \& Fouladkhah, A. Outbreak History, Biofilm Formation, and Preventive Measures for Control of Cronobacter sakazakii in Infant Formula and Infant Care Settings. Microorganisms 7, 77 (2019).

8. WHO (World Health Organization) and Food and Agriculture Organization of the United Nations. Safe preparation, storage and handling of powdered infant formula. Guidelines. (2007). 
9. Agostoni, C. et al. Preparation and handling of powdered infant formula: a commentary by the ESPGHAN Committee on Nutrition. J. Pediatr. Gastroenterol. Nutr. 39, 320-322 (2004).

10. Official Journal of the European Union. COMMISSION REGULATION (EC) No 2073/2005 of 15 November 2005 on microbiological criteria for foodstuffs. (2005).

11. AFSSA. Décision du 3 décembre 2007 définissant les règles de bonnes pratiques prévues à l'alinéa 3 de l'article L. 2323-1 du code de la santé publique. (2007).

12. CLIN Paris-Nord. Alimentation du nourrisson en collectivité pédiatrique: conditions de préparation et circuit des biberons. (1999).

13. Official Journal of the European Union. COUNCIL DIRECTIVE 93/43/EEC of 14 June 1993 on the hygiene of foodstuffs. (1993).

14. Légifrance. Arrêté du 29 septembre 1997 fixant les conditions d'hygiène applicables dans les établissements de restauration collective à caractère social. (1997). 


\section{Annexes}

Annexe $\mathbf{n}^{\circ} 1$ : Questionnaire soumis aux biberonneries

\section{Organisation structurelle :}

Depuis quand possédez-vous une biberonnerie?

Possédez-vous une biberonnerie unique délivrant le lait pour l'ensemble des services de pédiatrie, ou chaque service possède t-il sa propre biberonnerie ?

Votre biberonnerie se situe t-elle à proximité des services de biberonnerie ?

La structure des locaux respecte-t-elle le principe de la marche en avant ?

\section{Responsabilités et ressources humaines :}

Sous la responsabilité de quel service est placée votre biberonnerie?

Qui est le référent médical de votre biberonnerie ? Quelles sont ses missions ?

Qui est le référent paramédical de votre biberonnerie? Quelles sont ses missions?

Vos agents sont-ils dédiés et formés spécifiquement pour assurer le fonctionnement de la biberonnerie?

Qui est autorisé à réaliser les prescriptions de lait?

\section{Stockage et hygiène :}

Où le lait est-il stocké ?

Quelle est la durée maximale de stockage?

Le matériel de stockage est-il désinfecté régulièrement?

La température est-elle tracée ?

Dans quelques conditions le lait est-il acheminé aux unités de soins ?

\section{Contrôles bactériologiques :}

Par qui les contrôles bactériologiques sont-ils réalisés ?

Quel référentiel utilisez-vous?

A quel intervalle sont-ils réalisés ?

Quels sont les germes recherchés ?

\section{Démarche qualité :}

Disposez-vous d'un système informatisé de gestion de la biberonnerie ?

Avez-vous mis en place un système de contrôle qualité au sein de votre biberonnerie ?

Qui en est le responsable et quelles sont ses qualifications?

Des protocoles sont-ils rédigés pour chaque tache relevant de la biberonnerie ?

Ces protocoles sont-ils accessibles à tous?

Mettez-vous en place des actions correctives suite à ces contrôles ? 
$\underline{\text { Annexe } \mathbf{n}^{\circ} 2 \text { : }}$ Organigramme de la biberonnerie du CHU Amiens-Picardie

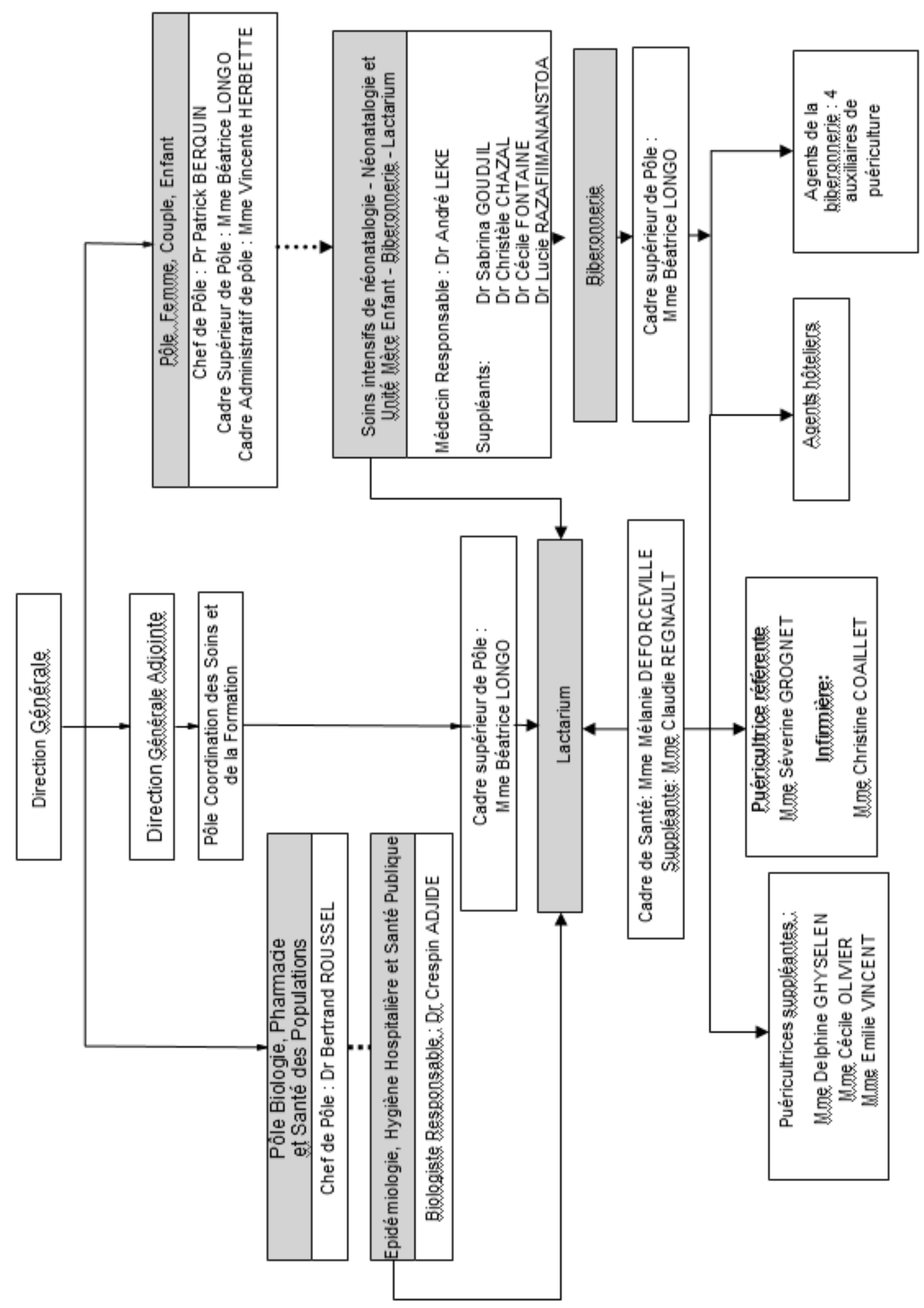




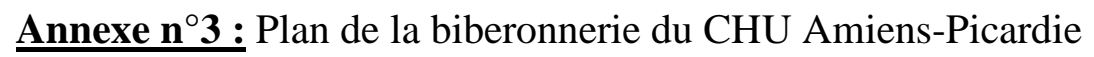

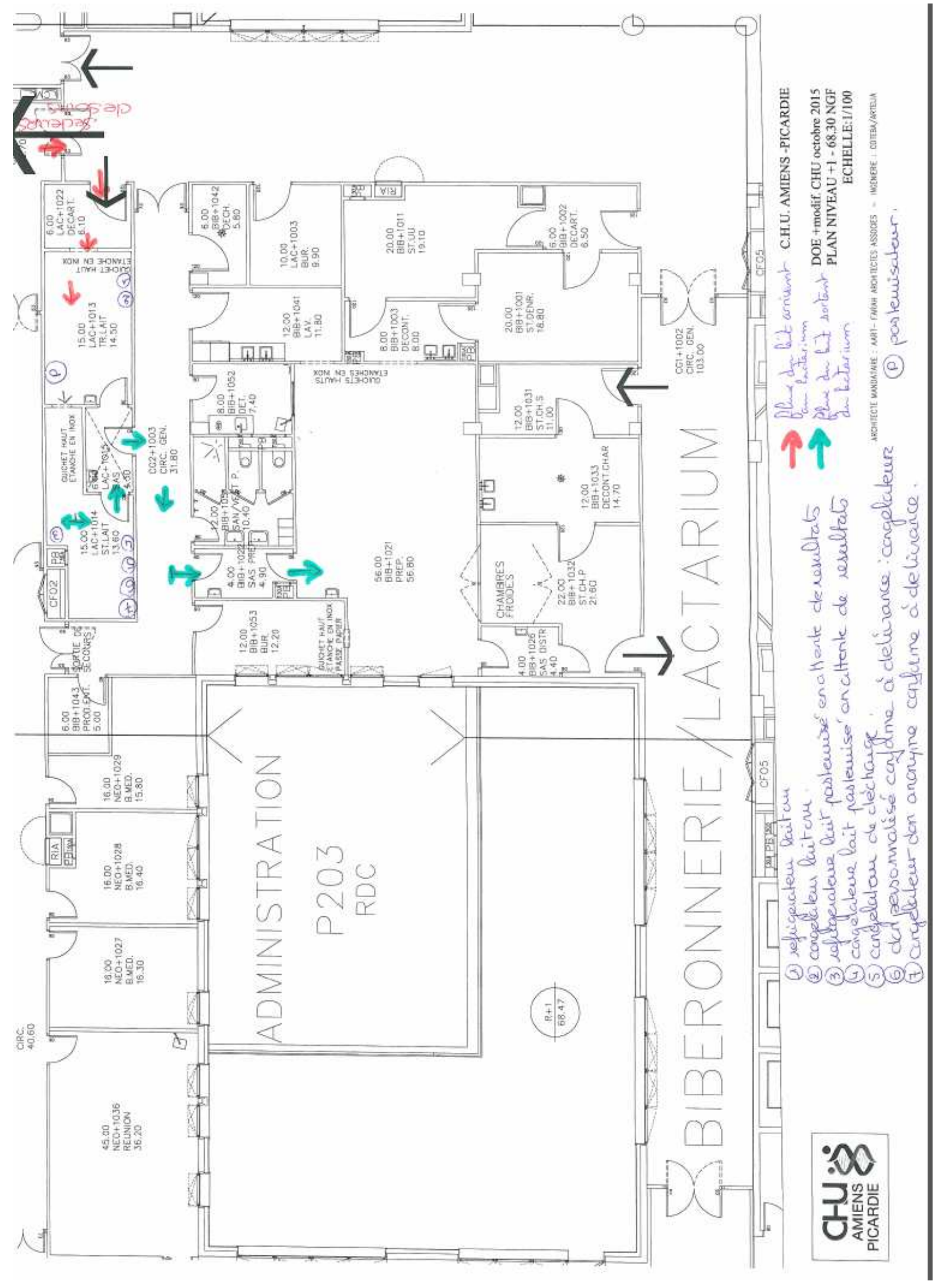




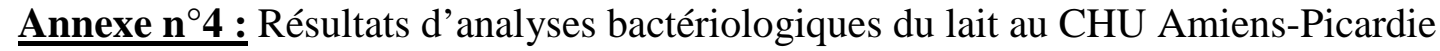

RAPPORT D'ALERTE

\begin{tabular}{|lll|}
\hline $\begin{array}{l}\text { Contact role } \\
\text { Code établissement }\end{array}$ & CHUAMM-01 & \\
Référence rapport & CHUAM-8012 & \\
Emis le 15/07/2019 & & Page 1/1 \\
\hline
\end{tabular}

Analysé au laboratoire de Merville

Zl du petit pacaux - Rue de l'aérodrome 59660 Merville

Service relation clients : supportrdemxns.com

Tel:0820 425435 (Service 0,12€/min+prix appel) Fax:013441 1335

Adresse de visite : HOPITAL-BIBERONNERIE:APRES CONSERV.24H

. PLACE VICTOR PAUCHET 80000 AMIENS

France

CHU AMIENS BIBERONNERIE

Dr LEKE Andre

. PEDIATRIE II

PLACE VICTOR PAUCHET

80054 AMIENS CEDEX 1

France

Référence échantillon 20864511 / Code prélèvement 119792079

Program Product AB11 / Code produit 116969 / Critére RSCHA01

DESCRIPTIF ÉCHANTILLON_Les informations en italique sont fournies par le client.

Nature de léchantillon: CONFORT

Stade de prélèvement

FAB SUR PLACE OUNÉG MANIP

STADE VENTE (RD)

Atmosphère

AUTRES

Date de prélèvement

Heure de prélèvement

$10 / 07 / 2018$

$T\left({ }^{\circ} \mathrm{C}\right)$ produit

17:05

5

Date de réception labo

$\mathrm{T}\left({ }^{\circ} \mathrm{C}\right)$ de réception

$11 / 07 / 2019$

Date de mise en analyse

4,3

$11 / 07 / 201916: 53$

PRÉLEVÉ PAR LE CLIENT ET

COLLECTE PAR SILLIKER

Etat physique

Intervention opérateur

Cadre de prélevement

Lieu de prélèvement

Condition de prélèvement

Contenant

Date de préparation

Identification de lot

DLUO

\section{을 MERIEUX}

Realisation prélevement

\section{FRAIS}

PREPARATION SUR PLACE

AUTOCONTROLE

ARMOIRE REFRIGEREE

COLLECTE

BOUTEILLE PLASTIQUE

09/07/2019

1008473843501

$24 / 08 / 2020$

\section{COMPTE RENDU D'ALERTE}

\begin{tabular}{|c|c|c|c|c|}
\hline \multicolumn{5}{|l|}{ COMPTE RENDU D'ALERTE } \\
\hline Essais & Résultats & Unités & Valeurs de référence & Sources \\
\hline $\begin{array}{l}\text { Micro-organismes aérobies } 30^{\circ} \mathrm{C} \\
\text { NF EN ISO } 4833-1\end{array}$ & $<1000$ & ufclg & 50000 & \\
\hline $\begin{array}{l}\text { Bacillus cereus présomptif } 30^{\circ} \mathrm{C} \\
\text { NF EN ISO } 7932\end{array}$ & $<10$ & ufc/g & 50 & \\
\hline $\begin{array}{l}\text { Recherche de Salmonella spp } \\
\text { BKR 2307-10/11 }\end{array}$ & Non Détecté & $725 \mathrm{~g}$ & Non Détecté & \\
\hline $\begin{array}{l}\text { Recherche d'Enterobacteries } 30^{\circ} \mathrm{C} \\
\text { NF EN ISO 21528-1 }\end{array}$ & Suspicion & $/ 10 \mathrm{~g}$ & Non Détecté & \\
\hline \multicolumn{5}{|l|}{ Alerte: 1802715 MC-SP-020 Dispositions CDC } \\
\hline $\begin{array}{l}\text { Staphylocoques à coagulase positive } 37^{\circ} \mathrm{C} \\
\text { NF EN ISO } 6888-2\end{array}$ & $<1 \#$ & ufc/g & 1 & \\
\hline $\begin{array}{l}\text { Clostridium perfringens } 37^{\circ} \mathrm{C} \\
\text { NF EN ISO } 7937\end{array}$ & $<10$ & ufclg & 10 & \\
\hline
\end{tabular}

Référence : CRITERE SELON CAHIER DES CHARGES CLIENT

= Anayge rtalate sur une boite - une dlution (methode inteme).

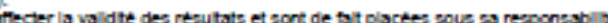

Seul le rapport d'essai définitif signé fait foi.

Seuls les résultats disponibles sont affichés.

Silliker SAS - Mérieux NutriSciences

25. Boulevard de la Paix - CS 38512 CERGY

95891 CERGY-PONTOISE CEDEX

Tél.: 0820562030 (Service 0.12€/min + prix appel) Fax: 0134411311 


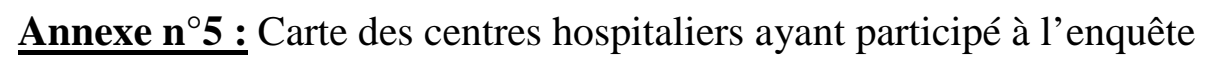

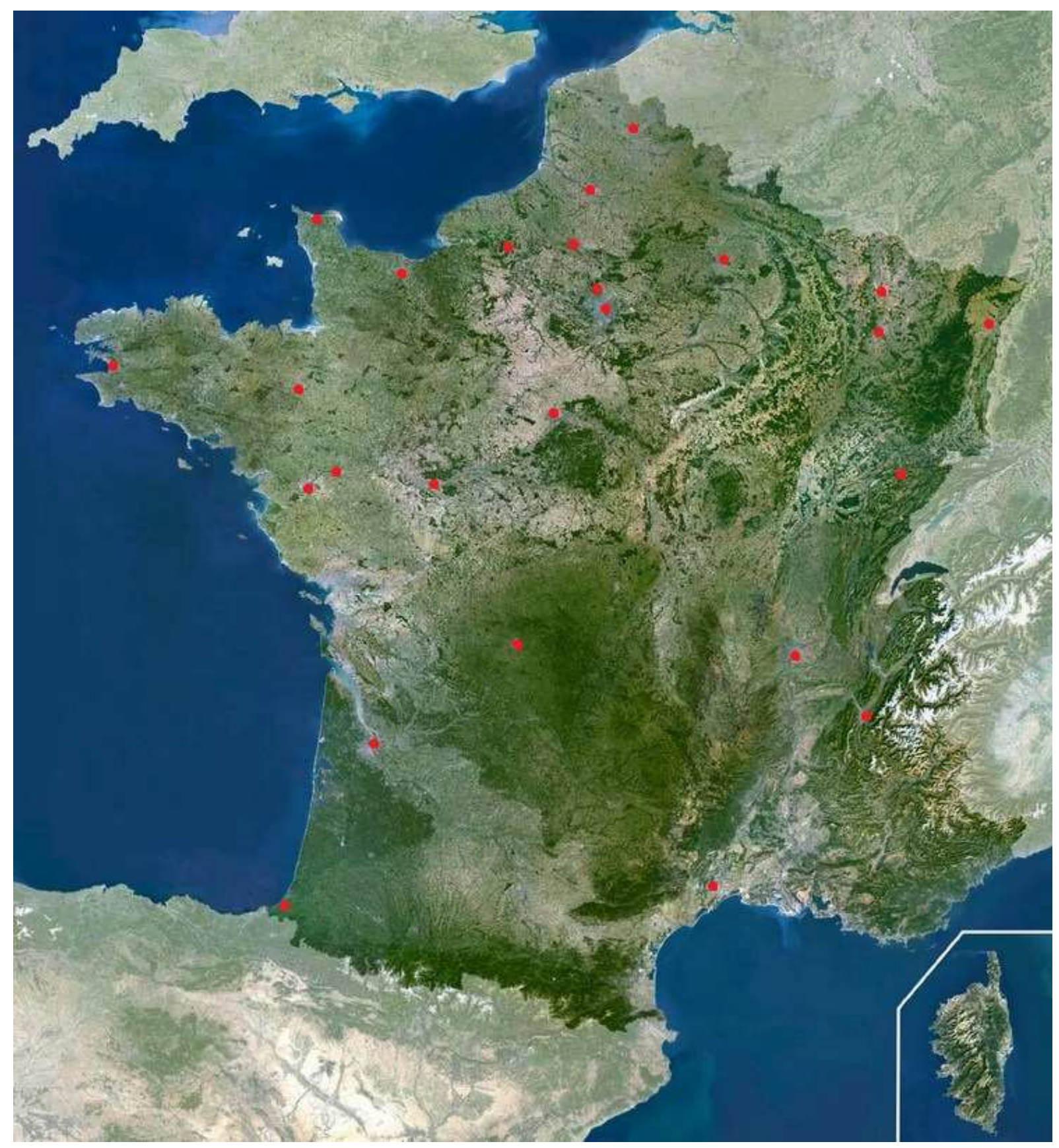




\section{Résumé}

Objectif : Le pédiatre joue un rôle essentiel pour assurer la sécurité alimentaire des jeunes enfants en participant à la veille sanitaire et en signalant toute infection qui pourrait être secondaire à une contamination bactérienne des préparations lactées. L'objectif principal de notre étude est d'évaluer le respect des recommandations de l'AFSSA émises en 2005.

Matériel et méthode : Un questionnaire a été soumis en ligne aux biberonneries des Centres hospitaliers Universitaires (CHU) et Centres Hospitaliers Généraux afin d'évaluer l'organisation structurelle et fonctionnelle de ces services, le respect des règles de préparation et de conservation des substituts du lait maternel et l'organisation des contrôles bactériologiques.

Résultats : 26 biberonneries ont répondu à notre étude. Les responsabilités médicales et paramédicales au sein des biberonneries sont parfaitement établies. La structure des locaux, de type biberonnerie unique en quasi-totalité, respecte le principe de la marche en avant. Les règles d'hygiène alimentaire sont appliquées par un personnel formé régulièrement. La démarche qualité est globalement mise en place avec une informatisation qui se généralise progressivement. En revanche, la manière dont sont organisés les contrôles bactériologiques sur les préparations infantiles est très différente d'un centre à l'autre, les recommandations ne fixant pas de règles claires contrairement à la réglementation appliquée aux lactariums.

Conclusion : Notre enquête nous montre que la réglementation actuelle ne permet pas une harmonisation des contrôles bactériologiques au sein des biberonneries. Cette harmonisation est une condition essentielle pour assurer une sécurité nutritionnelle maximale à nos patients les plus fragiles.

Mots-clés : biberonnerie, préparations infantiles, hygiène, contrôles bactériologiques, démarche qualité 


\begin{abstract}
Objective: The pediatrician plays a key role in ensuring the food safety of young children by participating in health monitoring and reporting any infection that may be secondary to bacterial contamination of the formula. The main objective of our study is to evaluate the respect of the recommendations of the AFSSA issued in 2005.

Methods: A form was submitted online to the formula rooms of the University Hospital Centers (CHU) and General Hospital Centers in order to evaluate the structural and functional organization of these services, the respect of the rules of preparation and conservation of the substitutes of the breast milk and the organization of bacteriological controls.

Results: 26 formula rooms answered to the study. The medical and paramedical responsibilities within the formula rooms are perfectly established. The structure of the premises, almost all singular formula rooms, respects the principle of walking forward. Food safety rules are applied by regularly trained staff. The quality approach is generally put in place with computerization that is gradually being generalized. On the other hand, the way in which bacteriological controls are organized on infant formulas is very different from one center to another, as the recommendations do not lay down clear rules, unlike the regulations applied to lactariums.

Conclusion: Our study shows that the current recommendations do not allow a harmonization of bacteriological controls within formula rooms in hospital. This harmonization is an essential condition to ensure maximum food safety for our most fragile patients.

Keywords: formula room, infant formula, food safety, bacteriological controls, quality approach
\end{abstract}

